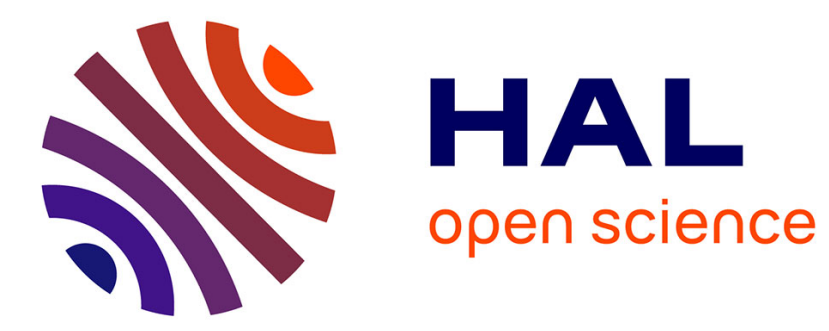

\title{
Low-order reconstruction operators on polyhedral meshes: application to compatible discrete operator schemes
}

\author{
Jerome Bonelle, Daniele Di Pietro, Alexandre Ern
}

\section{To cite this version:}

Jerome Bonelle, Daniele Di Pietro, Alexandre Ern. Low-order reconstruction operators on polyhedral meshes: application to compatible discrete operator schemes. Computer Aided Geometric Design, 2015, 35-36, pp.27-41. 10.1016/j.cagd.2015.03.015 . hal-01097311

\section{HAL Id: hal-01097311 https://hal.science/hal-01097311}

Submitted on 6 Jan 2015

HAL is a multi-disciplinary open access archive for the deposit and dissemination of scientific research documents, whether they are published or not. The documents may come from teaching and research institutions in France or abroad, or from public or private research centers.
L'archive ouverte pluridisciplinaire $\mathbf{H A L}$, est destinée au dépôt et à la diffusion de documents scientifiques de niveau recherche, publiés ou non, émanant des établissements d'enseignement et de recherche français ou étrangers, des laboratoires publics ou privés. 


\title{
Low-order reconstruction operators on polyhedral meshes: Application to Compatible Discrete Operator schemes
}

\author{
Jerome Bonelle \\ EDF R\&D \\ 6, quai Watier, BP 49 \\ 78401 Chatou Cedex, France \\ jerome.bonelle@edf.fr
}

\author{
Daniele A. Di Pietro \\ Université de Montpellier 2, I3M \\ I3M \\ 34057 Montpellier Cedex 5, France \\ daniele.di-pietro@univ-montp2.fr
}

\author{
Alexandre Ern \\ Université Paris-Est, CERMICS (ENPC) \\ Ecole des Ponts ParisTech \\ 77455 Marne la Vallée Cedex 2, France \\ ern@cermics.enpc.fr
}

December 19, 2014

\begin{abstract}
We study low-order reconstruction operators on polyhedral meshes, providing a unified framework for degrees of freedom attached to vertices, edges, faces, and cells. We present two equivalent sets of design properties and draw links with the literature. In particular, the two-level construction based on a $\mathbb{P}_{0^{-}}$ consistent and a stabilization part provides a systematic way of designing these operators. We present a simple example of piecewise constant reconstruction in each mesh cell, relying on geometric identities to fulfill the design properties on polyhedral meshes. Finally, we use these reconstruction operators to define a Hodge inner product and build Compatible Discrete Operator schemes, and we test the influence of the reconstruction operators in terms of accuracy and computational efficiency on an anisotropic diffusion problem.
\end{abstract}

\section{Introduction}

Reconstruction (or lifting) operators map degrees of freedom (DoFs) to functions living in a finite-dimensional space. DoFs are generally attached to some geometric entities of an underlying three-dimensional mesh, e.g., vertices, edges, faces, and cells. Reconstruction operators provide a right inverse of the de Rham (or reduction) operators which classically map fields (referred to as potentials, circulations, fluxes, and densities - or $k$-forms with $k \in\{0,1,2,3\}$ in the language of differential geometry) to DoFs attached to vertices, edges, faces, and cells, respectively. A reconstruction operator composed with the corresponding de Rham operator yields an interpolation operator. The reconstruction operator is said to be of low-order when this interpolation operator leaves cell-wise constant fields invariant.

Reconstruction operators are found in many applications. Our main focus here is the construction of discrete Hodge operators in the context of the numerical approximation of partial differential equations (PDEs). The discrete Hodge operator is the cornerstone of many compatible discretization schemes aiming at preserving properties of the PDE at the discrete level; see, e.g., Auchmann \& Kurz (2006); Bochev \& Hyman (2005); Bonelle (2014); Bonelle \& Ern (2014a); Bossavit (1988); Desbrun et al. (2005); Gerritsma (2012); Gillette \& Bajaj (2011); Hiptmair (2001); Tarhasaari et al. (1999); Teixeira (2001) and references therein. Many of these discretizations draw links between vector calculus, differential geometry, and algebraic topology. Reconstruction operators also constitute a powerful tool to analyze numerical schemes and to derive improved error estimates in different norms; see for instance Brezzi et al. (2005); Bonelle \& Ern (2014a); Di Pietro \& Lemaire (2015). One recent example is provided by the Compatible Discrete Operator (CDO) schemes for diffusive PDEs and the Stokes equations (Bonelle, 2014; Bonelle \& Ern, 2014a,b). To some extent, CDO schemes can be seen as an extension to polyhedral meshes of Discrete Exterior Calculus (DEC) schemes of Desbrun et al. (2005); Hirani (2003).

Reconstruction operators have been devised in the Finite Element (FE) literature for specific shapes of mesh cells (tetrahedron, hexahedron, pyramid...). The most famous examples are Whitney reconstruction functions in simplices (see Whitney, 1957). These reconstruction functions are built using the Courant hat functions for potentials, the (lowest-order) Nédélec shape functions for circulations, and the (lowest-order) Raviart-Thomas-Nédélec shape functions for fluxes. A typical way to extend the reconstruction of potentials 
to polyhedral meshes is to use the concept of generalized barycentric coordinates; see Floater et al. (2005); Gillette \& Bajaj (2011); Gillette et al. (2012); Hormann \& Sukumar (2008); Wachspress (1975); Warren et al. (2007) and references therein.

A generic way of building reconstruction operators for any type of DoFs on polyhedral meshes has been proposed by Brezzi et al. (2014); Christiansen (2008); Gillette et al. (2014). In both cases, the reconstruction operators are built locally in each mesh cell in such a way that suitable matching conditions are satisfied at mesh interfaces. Specifically, reconstructed potentials are continuous across interfaces, the tangential component of circulations is continuous, and so is the normal component of fluxes. Such matching conditions ensure the conformity of the reconstruction, in the sense that the operator maps to the appropriate Sobolev space such as $H^{1}(\Omega), H(\underline{\operatorname{curl}} ; \Omega)$, or $H(\operatorname{div} ; \Omega)$, where $\Omega$ is the computational domain discretized by the three-dimensional polyhedral mesh. The conformity of the reconstruction then plays a central role in the analysis of the numerical scheme.

An alternative viewpoint, not aiming at conformity, has been developed in the context of other discretization methods such as, e.g., the Hybrid Finite Volume (HFV) scheme by Eymard et al. (2010), the Discrete Geometric Approach (DGA) by Codecasa et al. (2010), and, more recently, the CDO schemes by Bonelle (2014); Bonelle \& Ern (2014a,b), the generalized Crouzeix-Raviart method by Di Pietro \& Lemaire (2015), and the Hybrid HighOrder (HHO) methods by Di Pietro \& Ern (2015); Di Pietro et al. (2014) (which also include the possibility to increase the approximation order). For the low-order schemes, the reconstruction operators typically map onto piecewise constant functions on a submesh (thereby discarding local conformity), while their composition with the de Rham operator remains single-valued. In this context, the analysis of the numerical schemes generally hinges on a novel property of the reconstruction, to which we refer as dual consistency.

In this paper, we devise low-order reconstruciton operators on polyhedral meshes within a unified framework for DoFs attached to vertices, edges, faces, and cells. A salient contribution is that we identify the design principles that reconstruction operators have to verify so that the resulting discrete Hodge operator satisfies $\mathbb{P}_{0^{-}}$ consistency and stability properties, which in turn ensure the convergence of the numerical scheme. Specifically, we establish the equivalence between a one-level design strategy of the reconstruction operator as considered in Codecasa et al. (2010); Bonelle \& Ern (2014a) and a two-level design strategy as considered in Brezzi et al. (2007); Di Pietro \& Ern (2013); Eymard et al. (2010). This second strategy, which decomposes the reconstruction operator into the sum of a consistent part and a stabilization part, provides a systematic construction principle where the only user-dependent design parameter is the weighting of the least-squares penalty underlying the stabilization part, while the consistent part is fixed. As an illustration, we present piecewise constant reconstruction operators in each mesh cell for all types of DoFs and study the impact in terms of accuracy and computational cost when varying the stabilization part of the reconstruction operators in the context of the numerical approximation of anisotropic diffusion problems on polyhedral meshes.

This paper is organized as follows. In Section 2, we introduce the different geometric entities. In Section 3 , we briefly present the CDO framework. in Section 4, we state the design properties of reconstruction operators on polyhedral cells, and show that the one- and two-level design principles are equivalent. In Section 5, we design a family of reconstruction operators which are piecewise constant on each mesh cell and which fulfill the design properties stated in Section 4. Finally, in Section 6, we present an application to CDO schemes for the approximation of anisotropic diffusion problems on polyhedral meshes.

\section{Geometric objects}

\subsection{Mesh and geometric entities}

The starting point is a discretization of the geometric domain $\Omega \subset \mathbb{R}^{3}$ by a (primal) mesh $\mathrm{M}:=\{\mathrm{V}, \mathrm{E}, \mathrm{F}, \mathrm{C}\}$ where $\mathrm{V}$ collects vertices (or 0-cells), E edges (or 1-cells), F faces (or 2-cells), and $\mathrm{C}$ cells (or 3-cells). A generic element of $\mathrm{V}$ (resp. E, F, C) is a vertex denoted by v (resp. an edge e, a face f, a cell c); see Figure 1. The mesh $\mathrm{M}$ has the structure of a cellular complex, in the sense that the boundary of a $k$-cell in $\mathrm{M}, 1 \leq k \leq 3$, can be decomposed into $(k-1)$-cells belonging to $\mathrm{M}$ (see Christiansen (2008)).

Let $A$ be any set among $\mathrm{V}, \mathrm{E}, \mathrm{F}$, or $\mathrm{C}$. If $\mathrm{x}$ is a geometric entity of $\mathrm{M}$ of dimension larger than that of the elements of $A$, we denote by $A_{\mathrm{x}}$ the subset defined by

$$
A_{\mathrm{x}}:=\{a \in A \mid a \subset \partial \mathrm{x}\}
$$




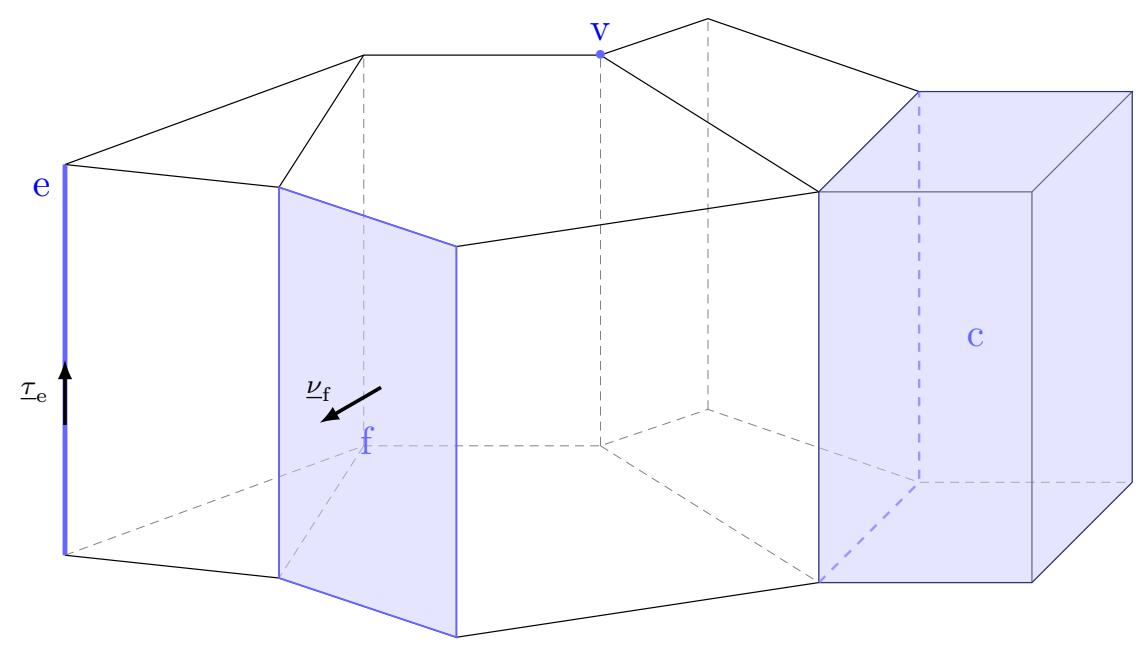

Figure 1: Example of primal mesh $M$ highlighting a vertex $v \in V$, an edge $e \in E$, a face $f \in F$, and a cell $c \in \mathrm{C}$.

otherwise,

$$
A_{\mathrm{x}}:=\{a \in A \mid \mathrm{x} \subset \partial a\} .
$$

For instance, $\mathrm{E}_{\mathrm{c}}:=\{\mathrm{e} \in \mathrm{E} \mid \mathrm{e} \subset \partial \mathrm{c}\}$ collects the edges of $\mathrm{c}$ and $\mathrm{C}_{\mathrm{e}}:=\{\mathrm{c} \in \mathrm{C} \mid \mathrm{e} \subset \partial \mathrm{c}\}$ collects the cells of which e is an edge. In what follows, design properties are stated on each cell $\mathrm{c} \in \mathrm{C}$. Therefore, the sets $\mathrm{V}_{\mathrm{c}}$, $\mathrm{E}_{\mathrm{c}}, \mathrm{F}_{\mathrm{c}}$, and $\mathrm{C}_{\mathrm{c}}$ play a key role (note that $\mathrm{C}_{\mathrm{c}}=\{\mathrm{c}\}$ ).

We often denote by $\mathrm{X}$ any set such as $\mathrm{V}, \mathrm{E}, \mathrm{F}$, or $\mathrm{C}$ and by $\mathrm{x}$ any geometric entity such as $\mathrm{v}$, e, f, or c. The cardinality of the set $\mathrm{X}$ is denoted by \#X.

Definition 1 (measure). $|\mathrm{x}|$ represents the measure of the entity $\mathrm{x}$. For a vertex $\mathrm{v} \in \mathrm{V},|\mathrm{v}|=1$ by convention, $|\mathrm{e}|$ is the length of the edge $\mathrm{e}, \mathrm{f} \mid$ is the area of the face $\mathrm{f}$, and $|\mathrm{c}|$ is the volume of the cell c.

Definition 2 (barycenter). The barycenters of an edge $\mathrm{e} \in \mathrm{E}$ and of a face $\mathrm{f} \in \mathrm{F}$ are defined, respectively, as follows:

$$
\underline{x}_{\mathrm{e}}:=\frac{1}{|\mathrm{e}|} \int_{\mathrm{e}} \underline{x} \quad \text { and } \quad \underline{x}_{\mathrm{f}}:=\frac{1}{|\mathrm{f}|} \int_{\mathrm{f}} \underline{x} .
$$

To each edge e $\in \mathrm{E}$, we arbitrarily assign a unit tangent vector $\underline{\tau}_{\mathrm{e}}$ and to each face f a unit normal vector $\underline{\nu}_{\mathrm{f}}$. Moreover, we define for all edges e $\in \mathrm{E}$ and all faces $\mathrm{f} \in \mathrm{F}$, respectively, the vectors

$$
\underline{\mathrm{e}}:=\int_{\mathrm{e}} \underline{\tau}_{\mathrm{e}}, \quad \underline{\mathrm{f}}:=\int_{\mathrm{f}} \underline{\nu}_{\mathrm{f}}
$$

Mesh assumption. We assume that all primal faces are planar and that each face $\mathrm{f} \in \mathrm{F}$ is star-shaped with respect to its barycenter. Moreover, we assume that each cell $\mathrm{c} \in \mathrm{C}$ is star-shaped with respect to a point $\underline{x}_{\bar{c}} \in \mathrm{c}$ (not necessarily the barycenter of c). In what follows, we denote by (MB) this set of assumptions.

\section{$2.2 \quad$ Geometric maps}

For the remaining part of the paper, we consider an arbitrary cell $\mathrm{c} \in \mathrm{C}$ and state definitions and properties for this cell.

Definition 3 (Primal geometric map). We introduce a primal geometric map $\mathfrak{g}_{\mathrm{X}_{\mathrm{c}}}: \mathrm{X}_{\mathrm{c}} \rightarrow \mathbb{E}_{\mathrm{X}}$, where $\mathbb{E}_{\mathrm{X}}$ corresponds to $\mathbb{R}$ if $\mathrm{X} \in\{\mathrm{V}, \mathrm{C}\}$ and to $\mathbb{R}^{3}$ if $\mathrm{X} \in\{\mathrm{E}, \mathrm{F}\}$, so that

$$
\begin{array}{ll}
\mathfrak{g}_{\mathrm{V}_{\mathrm{c}}}(\mathrm{v}):=1, & \forall \mathrm{v} \in \mathrm{V}_{\mathrm{c}}, \\
\mathfrak{g}_{\mathrm{E}_{\mathrm{c}}}(\mathrm{e}):=\underline{\mathrm{e}}, & \forall \mathrm{e} \in \mathrm{E}_{\mathrm{c}}, \\
\mathfrak{g}_{\mathrm{F}_{\mathrm{c}}}(\mathrm{f}):=\underline{\mathrm{f}}, & \forall \mathrm{f} \in \mathrm{F}_{\mathrm{c}}, \\
\mathfrak{g}_{\mathrm{C}_{\mathrm{c}}}(\mathrm{c}):=|\mathrm{c}| . &
\end{array}
$$



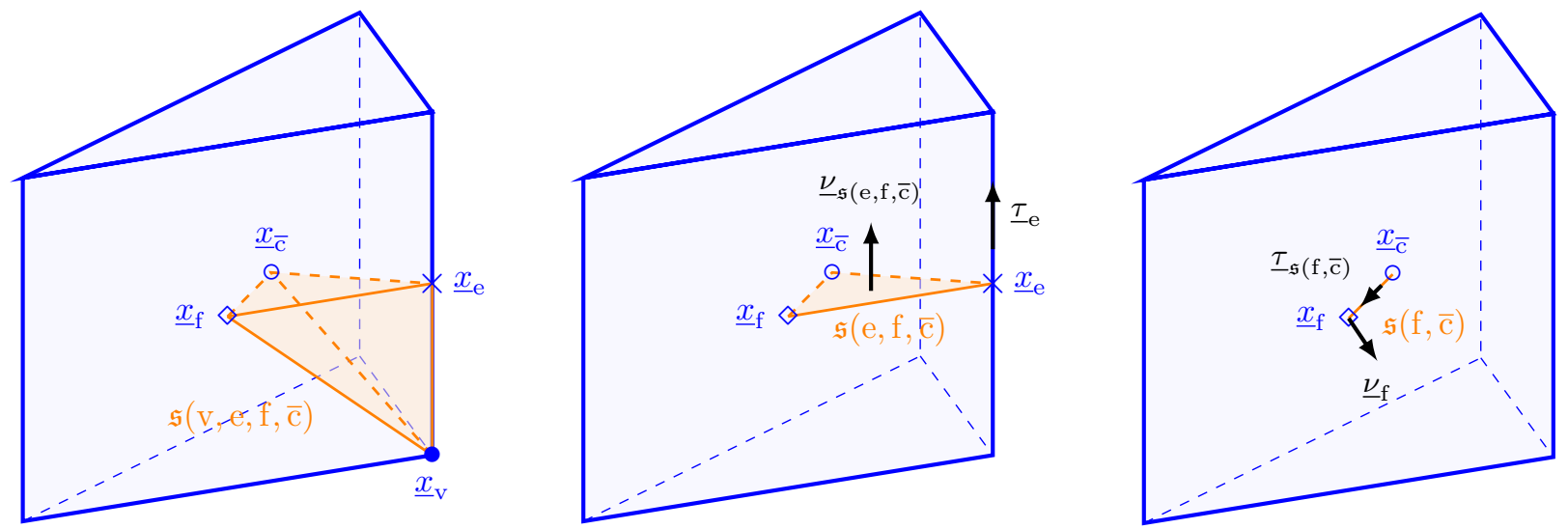

Figure 2: Example of a prismatic cell. Top left: the elementary tetrahedron $\mathfrak{s}(\mathrm{v}, \mathrm{e}, \mathrm{f}, \overline{\mathrm{c}})$ is highlighted; Top right: the elementary triangle $\mathfrak{s}(e, f, \overline{\mathrm{c}})$ is highlighted; Bottom: the elementary segment $\mathfrak{s}(\mathrm{f}, \overline{\mathrm{c}})$ is highlighted.

Definition 4 (simplex). For all $1 \leq k \leq 3$, given $(k+1)$ points $\left\{\underline{x}_{0}, \ldots, \underline{x}_{k}\right\}, \mathfrak{s}(0, \ldots, k)$ denotes the convex hull of these points (yielding, up to degenerate cases, a segment for $k=1$, a triangle for $k=2$, and a tetrahedron for $k=3)$; see Figure 2.

Definition 5 (Dual geometric map). We introduce a dual geometric map $\tilde{\mathfrak{g}}_{\mathrm{x}_{\mathrm{c}}}: \mathrm{X}_{\mathrm{c}} \rightarrow \mathbb{E}_{\mathrm{X}}$ defined as follows:

$$
\begin{array}{rlrl}
\tilde{\mathfrak{g}}_{\mathrm{V}_{\mathrm{c}}}(\mathrm{v}) & :=\sum_{\mathrm{e} \in \mathrm{E}_{\mathrm{v}} \cap \mathrm{E}_{\mathrm{c}} \sum_{\mathrm{f} \in \mathrm{F}_{\mathrm{e}} \cap \mathrm{F}_{\mathrm{c}}}|\mathfrak{s}(\mathrm{v}, \mathrm{e}, \mathrm{f}, \overline{\mathrm{c}})|,} & \forall \mathrm{v} \in \mathrm{V}_{\mathrm{c}} \\
\tilde{\mathfrak{g}}_{\mathrm{E}_{\mathrm{c}}}(\mathrm{e}):=\sum_{\mathrm{f} \in \mathrm{F}_{\mathrm{e}} \cap \mathrm{F}_{\mathrm{c}}}|\mathfrak{s}(\mathrm{e}, \mathrm{f}, \overline{\mathrm{c}})| \underline{\nu}_{\mathfrak{s}(\mathrm{e}, \mathrm{f}, \overline{\mathrm{c}})}, & \forall \mathrm{e} \in \mathrm{E}_{\mathrm{c}} \\
\tilde{\mathfrak{g}}_{\mathrm{F}_{\mathrm{c}}}(\mathrm{f}):=|\mathfrak{s}(\mathrm{f}, \overline{\mathrm{c}})| \underline{\tau}_{\mathfrak{s}(\mathrm{f}, \overline{\mathrm{c}})}, & \forall \mathrm{f} \in \mathrm{F}_{\mathrm{c}} \\
\tilde{\mathfrak{g}}_{\mathrm{C}_{\mathrm{c}}}(\mathrm{c}):=1 &
\end{array}
$$

where $\underline{\nu}_{\mathfrak{s}(\mathrm{e}, \mathrm{f}, \overline{\mathrm{c}})}$ is the unit normal vector to the triangle $\mathfrak{s}(\mathrm{e}, \mathrm{f}, \overline{\mathrm{c}})$ oriented according to $\underline{\tau}_{\mathrm{e}}$ for all faces $\mathrm{f} \in \mathrm{F}_{\mathrm{e}}$ and $\underline{\tau}_{\mathfrak{s}(\mathrm{f}, \overline{\mathrm{c}})}$ is the unit tangent vector to the segment $\mathfrak{s}(\mathrm{f}, \overline{\mathrm{c}})$ oriented according to $\underline{\nu}_{\mathrm{f}}$; see Figures 2 and 3.
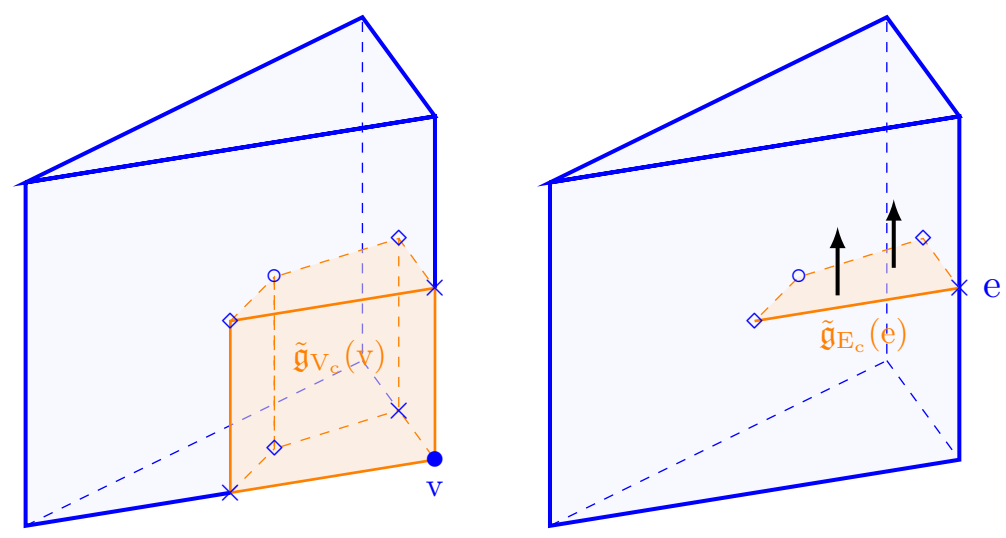

Figure 3: Example on a prismatic cell of the dual geometric map attached to a vertex v (left) and to an edge e (right).

Remark 6 (Dual mesh). The quantities specified in Definition 5 naturally appear when one considers a barycentric dual mesh. Namely, $\tilde{\mathfrak{g}}_{\mathrm{V}_{\mathrm{c}}}(\mathrm{v})$ is the volume of the dual cell associated with the vertex $\mathrm{v} \in \mathrm{V}_{\mathrm{c}}$, $\tilde{\mathfrak{g}}_{\mathrm{E}_{\mathrm{c}}}(\mathrm{e})$ is the vector area of the dual face associated with the edge $\mathrm{e} \in \mathrm{E}_{\mathrm{c}}$, and $\tilde{\mathfrak{g}}_{\mathrm{F}_{\mathrm{c}}}(\mathrm{f})$ is the vector length of the dual edge associated with the face $\mathrm{f} \in \mathrm{F}_{\mathrm{c}}$. 
Proposition 7 (magic formula). Assume (MB). Then, the following identity holds:

$$
\sum_{\mathrm{x} \in \mathrm{X}_{\mathrm{c}}} \tilde{\mathfrak{g}}_{\mathrm{x}_{\mathrm{c}}}(\mathrm{x}) \otimes \mathfrak{g}_{\mathrm{x}_{\mathrm{c}}}(\mathrm{x})=|\mathrm{c}| \mathbf{I d}_{\mathrm{X}} .
$$

If $\mathrm{X} \in\{\mathrm{V}, \mathrm{C}\}, \mathrm{Id}_{\mathrm{X}}$ is equal to 1 and $\otimes$ is simply a multiplication. If $\mathrm{X} \in\{\mathrm{E}, \mathrm{F}\}, \mathrm{Id}_{\mathrm{X}}$ is the $3 \times 3$ identity tensor and $\otimes$ is the tensor product.

Proof. The case $\mathrm{X} \in\{\mathrm{V}, \mathrm{C}\}$ is straightforward. The proof for the case $\mathrm{X} \in\{\mathrm{E}, \mathrm{F}\}$ is given in (Bonelle, 2014, Proposition 5.24); see also Codecasa \& Trevisan (2007).

The exact representation of constant fields by the reconstruction operators devised in this paper hinges on the identity (7).

\section{Overview of the CDO framework}

A detailed presentation of the CDO framework can be found in Bonelle (2014). In what follows, we only present the main ideas.

\subsection{Degrees of freedom}

Following the seminal ideas of Tonti (1975) and Bossavit (2000), DoFs are defined using de Rham maps, and their localization results from the physical nature of the fields. For a cell $\mathrm{c} \in \mathrm{C}$, the (local) finite dimensional space of DoFs related to discrete potentials is denoted by $\mathcal{V}_{\mathrm{c}}$ and collects the values of scalar fields at vertices,

$$
\left.\mathrm{R}_{\mathcal{V}_{\mathrm{c}}}(p)\right|_{\mathrm{v}}:=p\left(\underline{x}_{\mathrm{v}}\right), \quad \forall \mathrm{v} \in \mathrm{V}_{\mathrm{c}},
$$

that related to discrete circulations is denoted by $\mathcal{E}_{\mathrm{c}}$ and collects the integrals of the tangential component of vector fields along edges,

$$
\left.\mathrm{R}_{\mathcal{E}_{\mathrm{c}}}(\underline{g})\right|_{\mathrm{e}}:=\int_{\mathrm{e}} \underline{g} \cdot \underline{\tau}_{\mathrm{e}}, \quad \forall \mathrm{e} \in \mathrm{E}_{\mathrm{c}},
$$

that related to discrete fluxes is denoted by $\mathcal{F}_{\mathrm{c}}$ and collects the integrals of the normal component of vector fields across faces,

$$
\left.\mathrm{R}_{\mathcal{F}_{\mathrm{c}}}(\underline{\phi})\right|_{\mathrm{f}}:=\int_{\mathrm{f}} \underline{\phi} \cdot \underline{\nu}_{\mathrm{f}}, \quad \forall \mathrm{f} \in \mathrm{F}_{\mathrm{c}},
$$

and that related to discrete densities is denoted by $\mathcal{C}_{\mathrm{c}}$ and collects the integral of scalar fields over the cell,

$$
\left.\mathrm{R}_{\mathcal{C}_{\mathrm{c}}}(s)\right|_{\mathrm{c}}:=\int_{\mathrm{c}} s .
$$

Let $\mathcal{X}_{\mathrm{c}} \in\left\{\mathcal{V}_{\mathrm{c}}, \mathcal{E}_{\mathrm{c}}, \mathcal{F}_{\mathrm{c}}, \mathcal{C}_{\mathrm{c}}\right\}$. De Rham maps $\mathrm{R}_{\mathcal{X}_{\mathrm{c}}}: S_{\mathcal{X}}(\mathrm{c}) \rightarrow \mathcal{X}_{\mathrm{c}}$ act on sufficiently smooth fields so that DoFs are single valued. The domain of the de Rham maps can be taken to be, for instance, $S_{\mathcal{V}}(\mathrm{c})=H^{\frac{3}{2}+\delta}(\mathrm{c})$, $S_{\mathcal{E}}(\mathrm{c})=H^{1+\delta}(\mathrm{c}), S_{\mathcal{F}}(\mathrm{c})=H^{\frac{1}{2}+\delta}(\mathrm{c})$, and $S_{\mathcal{C}}(\mathrm{c})=L^{2}(\mathrm{c})$ with $\delta>0$. Moreover, $\mathbf{a} \in \mathcal{X}_{\mathrm{c}}$ can be viewed as an array of size $\# \mathrm{X}_{\mathrm{c}}$ since $\mathcal{X}_{\mathrm{c}}$ is isomorphic to $\mathbb{R}^{\# \mathrm{X}_{\mathrm{c}}}$. The value of the DoF attached to the entity $\mathrm{x} \in \mathrm{X}_{\mathrm{c}}$ is denoted by $\mathbf{a}_{\mathrm{x}} \in \mathbb{R}$.

Remark 8 (Link with algebraic topology). Elements of $\mathcal{V}_{\mathrm{c}}$ (resp. $\mathcal{E}_{\mathrm{c}}, \mathcal{F}_{\mathrm{c}}, \mathcal{C}_{\mathrm{c}}$ ) are 0-cochains (resp. 1-, 2-, 3-cochains).

\subsection{Reconstruction operators}

Definition 9 (Local reconstruction operator). Let $\mathrm{c} \in \mathrm{C}$. The local reconstruction operator $\mathrm{L}_{\mathcal{X}_{\mathrm{c}}}: \mathcal{X}_{\mathrm{c}} \rightarrow P_{\mathcal{X}}(\mathrm{c})$ is defined in terms of a family of $\# \mathrm{X}_{\mathrm{c}}$ linearly independent reconstruction functions $\left\{\ell_{\mathrm{x}, \mathrm{c}}\right\}_{\mathrm{x}} \in \mathrm{X}_{\mathrm{c}}$ spanning the finite-dimensional space $P_{\mathcal{X}}(\mathrm{c})$, called the approximation space, so that the reconstructed field $\mathrm{L}_{\mathcal{X}_{c}}(\mathbf{a})$ is defined by

$$
\mathrm{L}_{\mathcal{X}_{\mathrm{c}}}(\mathbf{a})(\underline{x}):=\sum_{\mathbf{x} \in \mathrm{X}_{\mathrm{c}}} \mathbf{a}_{\mathrm{x}} \ell_{\mathrm{x}, \mathrm{c}}(\underline{x}), \quad \forall \mathbf{a} \in \mathcal{X}_{\mathrm{c}}, \forall \underline{x} \in \mathrm{c} .
$$


The reconstruction functions $\ell_{\mathrm{x}, \mathrm{c}}$ take values in $\mathbb{E}_{\mathrm{X}}$ (scalar-valued for potential and density reconstructions, vector-valued for circulation and flux reconstrucitons). Wenever needed, we underline vector-valued functions and the corresponding reconstruction operators. The finite-dimensional space $P_{\mathcal{X}}(\mathrm{c})$ is for instance spanned by piecewise $\mathbb{E}_{\mathrm{X}}$-valued polynomials. We assume that the functions in $P_{\mathcal{X}}(\mathrm{c})$ are in the domain of the local de Rham map $\mathrm{R}_{\mathcal{X}_{\mathrm{c}}}$, i.e. $P_{\mathcal{X}}(\mathrm{c}) \subset S_{\mathcal{X}}(\mathrm{c})$.

\subsection{Discrete Hodge operators}

The name "Hodge operator" stems from a concept of differential geometry called the Hodge-star operator (see Frankel, 1997, Chapter 14, for instance). The Hodge operator embeds a metric (usually induced by a phenomenological parameter) and connects spaces in duality ( $k$-forms and $(d-k)$-forms where $d$ is the space dimension and $k$ an integer such that $0 \leq k \leq d)$. So, there are four distinct Hodge operators in a three-dimensional space. As its continuous analogue, a discrete Hodge operator is a metric operator since its definition relies on geometric quantities (lengths, areas, volumes...) and on the evaluation of a material property.

Hodge inner product. A discrete Hodge operator can be classically associated with a bilinear form which we call Hodge inner product in what follows.

Definition 10 (local Hodge inner product). Let $\mathcal{X}_{\mathrm{c}} \in\left\{\mathcal{V}_{\mathrm{c}}, \mathcal{E}_{\mathrm{c}}, \mathcal{F}_{\mathrm{c}}, \mathcal{C}_{\mathrm{c}}\right\}$. Let $\alpha$ denote a material property assumed to be cellwise constant, taking values in $\mathbb{E}_{\mathrm{X}} \otimes \mathbb{E}_{\mathrm{X}}$ (scalar-valued for potentials and densities and tensor-valued for circulations and fluxes), and symmetric positive definite. A local reconstruction operator $\mathrm{L}_{\mathcal{X}_{\mathrm{c}}}$ or, equivalently, a set of local reconstruction functions $\left\{\ell_{\mathrm{x}, \mathrm{c}}\right\}_{\mathrm{x} \in \mathrm{X}_{\mathrm{c}}}$ defines a local Hodge inner product as follows:

$$
\mathrm{H}_{\alpha}^{\mathcal{X}_{\mathrm{c}}}\left(\mathbf{a}_{1}, \mathbf{a}_{2}\right):=\int_{\mathrm{c}} \mathrm{L}_{\mathcal{X}_{\mathrm{c}}}\left(\mathbf{a}_{1}\right) \cdot \alpha \cdot \mathrm{L}_{\mathcal{X}_{\mathrm{c}}}\left(\mathbf{a}_{2}\right), \quad \forall \mathbf{a}_{1}, \mathbf{a}_{2} \in \mathcal{X}_{\mathrm{c}} .
$$

As previously noticed by Bossavit (2000) with the concept of Galerkin Hodge based on Whitney reconstruction functions, the algebraic realization of the Hodge inner product defined by (9) is the mass matrix of the local reconstruction functions weighted by the material property $\alpha$. Thus, the link between the CDO approach and the FE approach appears naturally since FE shape functions can be used to build reconstruction operators.

Design strategies. Discrete Hodge operators are the cornerstone of the CDO approach. Well-posedness, convergence and error estimates hinge on the properties satisfied by this operator (see Bonelle \& Ern, 2014a,b, for elliptic and Stokes problems respectively). In the CDO framework, the crucial point is thus the design of the discrete Hodge operator or, equivalently, the Hodge inner product. Each definition leads to a different scheme.

In the specific case of Cartesian or Delaunay-Voronoï meshes and an isotropic material property, a diagonal discrete Hodge operator can be build as noticed in the DEC schemes by Desbrun et al. (2006) or in the covolume schemes by Nicolaides (1992). In more general situations, it is possible to design a discrete Hodge operator whose algebraic realization is a sparse and symmetric positive definite (SPD) matrix. There are two main design strategies. Either one directly sets the entries of the matrix as in DEC or Mimetic Finite Difference (MFD) schemes (see Brezzi et al., 2005, 2009) or one relies on Definition 10 using reconstruction functions. In this paper, we focus on this second strategy.

Local design properties. Since a (global) Hodge inner product results from a cellwise assembly process, the design properties are stated locally, i.e. in each mesh cell. The design of the (local) Hodge inner product hinges on the two following properties:

(H1) Stability. There is a real number $\eta_{\alpha}>0$ possibly depending on $\alpha$ but uniform w.r.t. c such that for all $\mathbf{a} \in \mathcal{X}_{\mathrm{c}}$

$$
\eta_{\alpha}\|\mathbf{a}\|_{\mathcal{X}_{\mathrm{c}}}^{2} \leq \mathrm{H}_{\alpha}^{\mathcal{X}_{\mathrm{c}}}(\mathbf{a}, \mathbf{a}) \leq \eta_{\alpha}^{-1}\|\mathbf{a}\|_{\mathcal{X}_{\mathrm{c}}}^{2}
$$

where $\|\mathbf{a}\|_{\mathcal{X}_{\mathrm{c}}}^{2}:=\sum_{\mathrm{x} \in \mathrm{X}_{\mathrm{c}}}\left|\mathfrak{p}_{\mathrm{x}, \mathrm{c}}\right|\left(\frac{\left|\mathbf{a}_{\mathrm{x}}\right|}{|\mathrm{x}|}\right)^{2}$ and $\mathfrak{p}_{\mathrm{x}, \mathrm{c}}$ is a subvolume related to a partition of the cell (cf. Section 5.1 and Figure 4). For analysis purposes, $\left|\mathfrak{p}_{\mathrm{x}, \mathrm{c}}\right|$ may be replaced by any equivalent volume (e.g. $\left.|\mathrm{c}|\right)$. The only consequence is a modification of the value of $\eta_{\alpha}$. 
(H2) $\mathbb{P}_{0}$-consistency. For any constant field $K \in \mathbb{E}_{\mathrm{X}}$, the following identity holds for all a $\in \mathcal{X}_{\mathrm{c}}$ :

$$
\mathrm{H}_{\alpha}^{\mathcal{X}_{\mathrm{c}}}\left(\mathrm{R}_{\mathcal{X}_{\mathrm{c}}}(K), \mathbf{a}\right)=K \cdot \alpha \cdot\left(\sum_{\mathbf{x} \in \mathrm{X}_{\mathrm{c}}} \mathbf{a}_{\mathrm{x}} \tilde{\mathfrak{g}}_{\mathrm{x}_{\mathrm{c}}}(\mathrm{x})\right)
$$

\section{Design properties of recontruction operators}

The design of reconstruction operators aims at recovering the two local design properties $(\mathbf{H 1})$ and $(\mathbf{H 2})$ of the Hodge inner product. There are two equivalent approaches, hereafter called one-level and two-level approach. The one-level approach directly requires properties on the reconstruction operators (or functions), while the two-level approach considered a decomposition of the reconstruction operators (or functions) into a consistent and a stabilization part.

\subsection{One-level approach}

This approach is considered by Codecasa et al. (2010) (except for (R1), see Bonelle \& Ern (2014a)). We require that:

(R1) Stability. There exists a real number $\eta_{\mathcal{X}}>0$ uniform w.r.t. c such that for all $\mathbf{a} \in \mathcal{X}_{\mathrm{c}}$,

$$
\eta_{\mathcal{X}}\|\mathbf{a}\|_{\mathcal{X}_{\mathrm{c}}}^{2} \leq\left\|L_{\mathcal{X}_{\mathrm{c}}}(\mathbf{a})\right\|_{L^{2}(\mathrm{c})}^{2} \leq \eta_{\mathcal{X}}^{-1}\|\mathbf{a}\|_{\mathcal{X}_{\mathrm{c}}}^{2} .
$$

(R2) Partition of unity. For any constant field $K \in \mathbb{E}_{\mathrm{X}}$, the following identity holds:

$$
\mathrm{L}_{\mathcal{X}_{\mathrm{c}}} \mathrm{R}_{\mathcal{X}_{\mathrm{c}}}(K)=K \text {. }
$$

(R3) Dual consistency. The mean-value of $\mathrm{L}_{\mathcal{X}_{c}}$ satisfies the following identity:

$$
\int_{\mathrm{c}} \mathrm{L}_{\mathcal{X}_{\mathrm{c}}}(\mathbf{a})=\sum_{\mathrm{x} \in \mathrm{X}_{\mathrm{c}}} \mathbf{a}_{\mathrm{x}} \tilde{\mathfrak{g}}_{\mathrm{x}_{\mathrm{c}}}(\mathrm{x}), \quad \forall \mathbf{a} \in \mathcal{X}_{\mathrm{c}}
$$

(R4) Unisolvence. $\mathrm{L}_{\mathcal{X}_{\mathrm{c}}}$ is a right inverse of $\mathrm{R}_{\mathcal{X}_{\mathrm{c}}}$, i.e.

$$
\mathrm{R}_{\mathcal{X}_{\mathrm{c}}} \mathrm{L}_{\mathcal{X}_{\mathrm{c}}}(\mathbf{a})=\mathbf{a}, \quad \forall \mathbf{a} \in \mathcal{X}_{\mathrm{c}} .
$$

Proposition 11. If the Hodge inner product is built using (10), then the properties (R1)-(R3) imply (H1)(H2).

Proof. The stability property (H1) results from (R1) and (10) together with the positive-definiteness of $\alpha$. Let $K \in \mathbb{E}_{\mathrm{X}}$. Recall that $\alpha$ is constant in c. For all $\mathbf{a} \in \mathcal{X}_{\mathrm{c}},(\mathbf{H 2})$ results from

$$
\begin{array}{rlrl}
\mathrm{H}_{\alpha}^{\mathcal{X}_{\mathrm{c}}}\left(\mathrm{R}_{\mathcal{X}_{\mathrm{c}}}(K), \mathbf{a}\right) & =\int_{\mathrm{c}} \mathrm{L}_{\mathcal{X}_{\mathrm{c}}} \mathrm{R}_{\mathcal{X}_{\mathrm{c}}}(K) \cdot \alpha \cdot \mathrm{L}_{\mathcal{X}_{\mathrm{c}}}(\mathbf{a}) & \text { by }(9), \\
& =\int_{\mathrm{c}} K \cdot \alpha \cdot \mathrm{L}_{\mathcal{X}_{\mathrm{c}}}(\mathbf{a}) & & \text { by }(\mathbf{R 2}), \\
& =K \cdot \alpha \cdot \sum_{\mathbf{x} \in \mathrm{X}_{\mathrm{c}}} \mathbf{a}_{\mathrm{x}} \tilde{\mathfrak{g}}_{\mathrm{x}_{\mathrm{c}}}(\mathrm{x}) & & \text { by }(\mathbf{R 3}) .
\end{array}
$$

Therefore, every discrete Hodge operator built from (9) with a reconstruction operator verifying the three properties (R1)-(R3) inherits the properties (H1) and (H2), so that the theoretical results derived in (Bonelle, 2014, Chapter 6) and Bonelle \& Ern (2014a) hold.

Remark 12 (Unisolvence). Observe that the unisolvence property $(\mathbf{R 4})$ is not needed to satisfy $(\mathbf{H 1})$ and (H2). 
Local design properties on reconstruction functions. We now rewrite the properties $(\mathbf{R 2})-(\mathbf{R} 4)$ in terms of reconstruction functions for each type of DoFs. We only state the results since the proofs are straightforward.

Proposition 13 (Potential reconstruction functions).

$$
\begin{aligned}
&(\mathbf{R 2}) \Leftrightarrow \sum_{\mathrm{v} \in \mathrm{V}_{\mathrm{c}}} \ell_{\mathrm{v}, \mathrm{c}}(\underline{x})=1, \quad \forall \underline{x} \in \mathrm{c}, \\
&(\mathbf{R 3}) \Leftrightarrow \quad \int_{\mathrm{c}} \ell_{\mathrm{v}, \mathrm{c}}=\tilde{\mathfrak{g}}_{\mathrm{v}_{\mathrm{c}}}(\mathrm{v}), \quad \forall \mathrm{v} \in \mathrm{V}_{\mathrm{c}}, \\
&(\mathbf{R} 4) \Leftrightarrow \quad \ell_{\mathrm{v}, \mathrm{c}}\left(\underline{x}_{\mathrm{v}^{\prime}}\right)=\delta_{\mathrm{v}, \mathrm{v}^{\prime}}, \quad \forall \mathrm{v}, \mathrm{v}^{\prime} \in \mathrm{V}_{\mathrm{c}},
\end{aligned}
$$

where $\delta_{\bullet}, \bullet$ is the Kronecker symbol.

Proposition 14 (Circulation reconstruction functions).

$$
\begin{array}{rlrl}
(\mathbf{R 2}) & \Leftrightarrow \quad \sum_{\mathrm{e} \in \mathrm{E}_{\mathrm{c}}} \underline{\ell}_{\mathrm{e}, \mathrm{c}}(\underline{x}) \otimes \underline{\mathrm{e}}=\underline{\underline{\mathrm{Id}}}, & \forall \underline{x} \in \mathrm{c}, \\
(\mathbf{R 3}) \Leftrightarrow \int_{\mathrm{c}} \underline{\ell}_{\mathrm{e}, \mathrm{c}}=\tilde{\mathfrak{g}}_{\mathrm{E}_{\mathrm{c}}}(\mathrm{e}), & \forall \mathrm{e} \in \mathrm{E}_{\mathrm{c}}, \\
(\mathbf{R 4}) \Leftrightarrow \quad \int_{\mathrm{e}^{\prime}} \underline{\ell}_{\mathrm{e}, \mathrm{c}} \cdot{\underline{\tau^{\prime}}}_{\mathrm{e}^{\prime}}=\delta_{\mathrm{e}, \mathrm{e}^{\prime}}, & \forall \mathrm{e}, \mathrm{e}^{\prime} \in \mathrm{E}_{\mathrm{c}} .
\end{array}
$$

Proposition 15 (Flux reconstruction functions).

$$
\begin{aligned}
(\mathbf{R 2}) \Leftrightarrow \sum_{\mathrm{f} \in \mathrm{F}_{\mathrm{c}}} \underline{\ell}_{\mathrm{f}, \mathrm{c}}(\underline{x}) \otimes \underline{\mathrm{f}}=\underline{\underline{\mathrm{Id}}}, \quad \forall \underline{x} \in \mathrm{c}, \\
(\mathbf{R 3}) \Leftrightarrow \int_{\mathrm{c}} \underline{\ell}_{\mathrm{f}, \mathrm{c}}=\tilde{\mathfrak{g}}_{\mathrm{F}_{\mathrm{c}}}(\mathrm{f}), \quad \forall \mathrm{f} \in \mathrm{F}_{\mathrm{c}}, \\
(\mathbf{R 4}) \Leftrightarrow \quad \int_{\mathrm{f}^{\prime}} \underline{\ell}_{\mathrm{f}, \mathrm{c}} \cdot \underline{\mathrm{f}}_{\mathrm{f}^{\prime}}=\delta_{\mathrm{f}, \mathrm{f}^{\prime}}, \quad \forall \mathrm{f}, \mathrm{f}^{\prime} \in \mathrm{F}_{\mathrm{c}} .
\end{aligned}
$$

Remark 16 (Density reconstruction). $\mathrm{L}_{\mathcal{C}_{\mathrm{c}}}$ is derived from a single reconstruction function $\ell_{\mathrm{c}}$ since $\# \mathrm{C}_{\mathrm{c}}=1$. From property $(\mathbf{R 2})$, we infer that

$$
\ell_{\mathrm{c}}(\underline{x})=\frac{1}{|\mathrm{c}|}, \quad \forall \underline{x} \in \mathrm{c} .
$$

We easily verify that this definition is in agreement with (R3) (since $\int_{\mathrm{c}} \mathrm{L}_{\mathcal{C}_{\mathrm{c}}}(\mathbf{a})=\mathbf{a}_{\mathrm{c}} \int_{\mathrm{c}} \ell_{\mathrm{c}}=\mathbf{a}_{\mathrm{c}}$ ) and $(\mathbf{R} 4)$ (since $\left.\int_{\mathrm{c}} \ell_{\mathrm{c}}=1\right)$.

In the remaining part of this paper, we focus on the case $\mathcal{X} \in\{\mathcal{V}, \mathcal{E}, \mathcal{F}\}$, the case $\mathcal{X}=\mathcal{C}$ being straightforward.

Remark 17 (Physical dimension). Observe that the reconstruction functions $\ell_{\mathrm{v}, \mathrm{c}}$ are dimensionless, $\underline{\ell}_{\mathrm{e}, \mathrm{c}}$ scale as the reciprocal of a length, $\underline{\ell}_{\mathrm{f}, \mathrm{c}}$ scale as the reciprocal of a surface, and $\ell_{\mathrm{c}}$ as the reciprocal of a volume.

Remark 18 ( $\mathbb{P}_{1}$-consistency). Whenever the linear completeness property

$$
\sum_{\mathrm{v} \in \mathrm{V}_{\mathrm{c}}} \underline{x}_{\mathrm{v}} \ell_{\mathrm{v}, \mathrm{c}}(\underline{x})=\underline{x}, \quad \forall \underline{x} \in \mathrm{c},
$$

holds along with $(\mathbf{R 2})$, this induces a $\mathbb{P}_{1}$-consistency property. Indeed, any affine field $A$ in $\mathrm{c}$ verifies $\mathrm{L}_{\mathcal{V}_{\mathrm{c}}} \mathrm{R}_{\mathcal{V}_{\mathrm{c}}}(A)=$ $A$. The field $A$ can be written as $A(\underline{x}):=A\left(\underline{x}_{\mathrm{c}}\right)+\underline{G} \cdot\left(\underline{x}-\underline{x}_{\mathrm{c}}\right)$ with $\underline{G}$ constant in $\mathrm{c}$, so that $\mathrm{L}_{\mathcal{V}_{\mathrm{c}}} \mathrm{R}_{\mathcal{V}_{\mathrm{c}}}(A(\underline{x}))=$ $\sum_{\mathrm{v} \in \mathrm{V}_{\mathrm{c}}} A\left(\underline{x}_{\mathrm{v}}\right) \ell_{\mathrm{v}, \mathrm{c}}(\underline{x})=A\left(\underline{x}_{\mathrm{c}}\right)+\underline{G} \cdot\left(\underline{x}-\underline{x}_{\mathrm{c}}\right)=A(\underline{x})$. 


\subsection{Two-level approach}

The second approach operates a decomposition of the reconstruction operator $\mathrm{L}_{\mathcal{X}_{\mathrm{c}}}$ into a consistent part $\mathrm{C}_{\mathcal{X}_{\mathrm{c}}}$ and a stabilization part $\mathrm{S}_{\mathcal{X}_{\mathrm{c}}}$, so that

$$
\mathrm{L}_{\mathcal{X}_{\mathrm{c}}}:=\mathrm{C}_{\mathcal{X}_{\mathrm{c}}}+\mathrm{S}_{\mathcal{X}_{\mathrm{c}}},
$$

with consistent part $\mathrm{C}_{\mathcal{X}_{\mathrm{c}}}$ taking a constant value in $\mathbb{E}_{\mathrm{X}}$ defined as follows:

$$
\mathrm{C}_{\mathcal{X}_{\mathrm{c}}}(\mathbf{a}):=\frac{1}{|\mathrm{c}|} \sum_{\mathrm{x} \in \mathrm{X}_{\mathrm{c}}} \mathbf{a}_{\mathrm{x}} \tilde{\mathfrak{g}}_{\mathrm{x}_{\mathrm{c}}}(\mathrm{x}), \quad \forall \mathbf{a} \in \mathcal{X}_{\mathrm{c}}
$$

and a stabilization part $\mathrm{S}_{\mathcal{X}_{\mathrm{c}}}: \mathcal{X}_{\mathrm{c}} \rightarrow P_{\mathcal{X}}(\mathrm{c})$ which is the only user-dependent part in the reconstruction operators. Observe that definition (18) implies

$$
\mathrm{C}_{\mathcal{X}_{\mathrm{c}}} \mathrm{R}_{\mathcal{X}_{\mathrm{c}}}(K)=K, \quad \forall K \in \mathbb{E}_{\mathrm{X}}
$$

owing to (7). Similar decompositions to (17) have been considered in the context of MFD schemes (see Brezzi et al., 2005, 2007), for the reconstruction of gradients in the context of HFV schemes (see Eymard et al. (2010) and also (Agélas et al., 2010, Section 3.3)) and of the generalized Crouzeix-Raviart method (see Di Pietro \& Lemaire, 2015), and for the reconstruction of gradients and fluxes in the context of HHO schemes (see Di Pietro \& Ern, 2013; Di Pietro et al., 2014; Di Pietro \& Ern, 2015).

Local design properties. Since the consistent part of the reconstruction operator is defined by (18), the design properties are stated on $\mathrm{S}_{\mathcal{X}_{\mathrm{c}}}$ for all $\mathrm{c} \in \mathrm{C}$. In addition to $(\mathbf{R} \mathbf{1})$ and $(\mathbf{R 4})$, we require that:

$(\mathbf{R} 2 *)$ For any constant field $K \in \mathbb{E}_{\mathrm{X}}$,

$$
\mathrm{S}_{\mathcal{X}_{\mathrm{c}}} \mathrm{R}_{\mathcal{X}_{\mathrm{c}}}(K)=0
$$

$(\mathbf{R 3} *)$ For all $\mathbf{a} \in \mathcal{X}_{\mathrm{c}}$

$$
\int_{\mathrm{c}} \mathrm{S}_{\mathcal{X}_{\mathrm{c}}}(\mathbf{a})=0
$$

In terms of reconstruction functions, the translation of (17) is

$$
\ell_{\mathrm{x}, \mathrm{c}}(\underline{x}):=\ell_{\mathrm{x}, \mathrm{c}}^{\mathrm{Co}}(\underline{x})+\ell_{\mathrm{x}, \mathrm{c}}^{\mathrm{St}}(\underline{x}), \quad \forall \mathrm{x} \in \mathrm{X}_{\mathrm{c}}, \forall \underline{x} \in \mathrm{c},
$$

where $\ell_{\mathrm{x}, \mathrm{c}}^{\mathrm{Co}}$ and $\ell_{\mathrm{x}, \mathrm{c}}^{\mathrm{St}}$ are respectively the consistent and stabilization part of the reconstruction function. We infer from (18) that

$$
\ell_{\mathrm{x}, \mathrm{c}}^{\mathrm{Co}}(\underline{x}):=\frac{\tilde{\mathfrak{g}}_{\mathrm{x}_{\mathrm{c}}}(\mathrm{x})}{|\mathrm{c}|}, \quad \forall \mathrm{x} \in \mathrm{X}_{\mathrm{c}}, \forall \underline{x} \in \mathrm{c} .
$$

Moreover, $(\mathbf{R 2} *)$ and $(\mathbf{R 3} *)$ are equivalent to

$(\mathbf{R} 2 *)$

$$
\begin{aligned}
& \Leftrightarrow \quad \sum_{\mathrm{x} \in \mathrm{X}_{\mathrm{c}}} \mathfrak{g}_{\mathrm{x}_{\mathrm{c}}}(\mathrm{x}) \cdot \ell_{\mathrm{x}, \mathrm{c}}^{\mathrm{St}}(\underline{x})=0, \\
& \Leftrightarrow \quad \int_{\mathrm{c}} \ell_{\mathrm{x}, \mathrm{c}}^{\mathrm{St}}=0, \quad \forall \mathrm{x} \in \mathrm{X}_{\mathrm{c}} .
\end{aligned}
$$

Proposition 19 (Link between the two sets of properties). Let $\mathrm{L}_{\mathcal{X}_{\mathrm{c}}}=\mathrm{C}_{\mathcal{X}_{\mathrm{c}}}+\mathrm{S}_{\mathcal{X}_{\mathrm{c}}}$ with $\mathrm{C}_{\mathcal{X}_{\mathrm{c}}}$ defined by (18). Then, $(\mathbf{R 2} *)$ is equivalent to $(\mathbf{R 2})$ and $(\mathbf{R 3} *)$ to $(\mathbf{R 3})$.

Proof. (R2) readily results from $(\mathbf{R 2} *)$ and (19). Moreover, $(\mathbf{R 3} *)$ yields

$$
\int_{\mathrm{c}} \mathrm{L}_{\mathcal{X}_{\mathrm{c}}}(\mathbf{a})=\int_{\mathrm{c}} \mathrm{C}_{\mathcal{X}_{\mathrm{c}}}(\mathbf{a})=\sum_{\mathrm{x} \in \mathrm{X}_{\mathrm{c}}} \mathbf{a}_{\mathrm{x}} \tilde{\mathfrak{g}}_{\mathrm{X}_{\mathrm{c}}}(\mathrm{x}),
$$

so that (R3) holds. The converse statement is proven with similar arguments.

A straightforward consequence of Proposition 19 is that every Hodge inner product built using reconstruction operators such that (R1) holds, the consistent part is defined by (18), and the stabilization part satisfies properties $(\mathbf{R 2} *)$ and $(\mathbf{R 3} *)$ inherits the properties (H1) and (H2). 
Proposition 20 (Orthogonal decomposition). A reconstruction operator built using (17) yields a Hodge inner product verifying for all $\mathbf{a}_{1}, \mathbf{a}_{2} \in \mathcal{X}_{\mathrm{c}}$,

$$
\mathrm{H}_{\alpha}^{\mathcal{X}_{\mathrm{c}}}\left(\mathbf{a}_{1}, \mathbf{a}_{2}\right):=\int_{\mathrm{c}} \mathrm{C}_{\mathcal{X}_{\mathrm{c}}}\left(\mathbf{a}_{1}\right) \cdot \alpha \cdot \mathrm{C}_{\mathcal{X}_{\mathrm{c}}}\left(\mathbf{a}_{2}\right)+\int_{\mathrm{c}} \mathrm{S}_{\mathcal{X}_{\mathrm{c}}}\left(\mathbf{a}_{1}\right) \cdot \alpha \cdot \mathrm{S}_{\mathcal{X}_{\mathrm{c}}}\left(\mathbf{a}_{2}\right) .
$$

Proof. This is a consequence of $(\mathbf{R 3} *)$ and the fact that $\mathrm{C}_{\mathcal{X}_{\mathrm{c}}}$ maps onto constant fields in $\mathbb{E}_{\mathrm{X}}$.

The consistent part of the Hodge inner product is identical for all choices of the reconstruction operator and is equal, for all $\mathbf{a}_{1}, \mathbf{a}_{2} \in \mathcal{X}_{\mathrm{c}}$, to

$$
\mathrm{H}_{\alpha}^{\mathcal{X}_{\mathrm{c}}}\left(\mathbf{a}_{1}, \mathbf{a}_{2}\right):=\frac{1}{|\mathrm{c}|} \sum_{\mathrm{x} \in \mathrm{X}_{\mathrm{c}}} \sum_{\mathrm{x}^{\prime} \in \mathrm{X}_{\mathrm{c}}} \mathbf{a}_{1, \mathrm{x}} \mathbf{a}_{2, \mathrm{x}^{\prime}} \tilde{\mathfrak{g}}_{\mathrm{x}_{\mathrm{c}}}(\mathrm{x}) \cdot \alpha \cdot \tilde{\mathfrak{g}}_{\mathrm{X}_{\mathrm{c}}}\left(\mathrm{x}^{\prime}\right) .
$$

\section{Piecewise constant reconstruction operators}

The goal of this section is to give an example of reconstruction operators on polyhedral meshes. We reconstruct potential (resp. circulation, flux) fields from DoFs attached to vertices (resp. edges, faces) using a piecewise constant approximation in each mesh cell. $\mathrm{L}_{\mathcal{V}}, \underline{\mathrm{L}}_{\mathcal{E}}$, and $\underline{\mathrm{L}}_{\mathcal{F}}$ are nonconforming reconstruction operators which embrace as particular cases, the DGA reconstruction operators of Codecasa et al. (2010) and HFV reconstruction operators of Eymard et al. (2010). This class of reconstruction operators is attractive from an implementation viewpoint since reconstruction operators are explicitly defined, i.e. they are not the numerical solutions of local problems.

\subsection{Cell partitions}

We first define three partitions of a cell based on the simplicial subdivision introduced in Section 2.
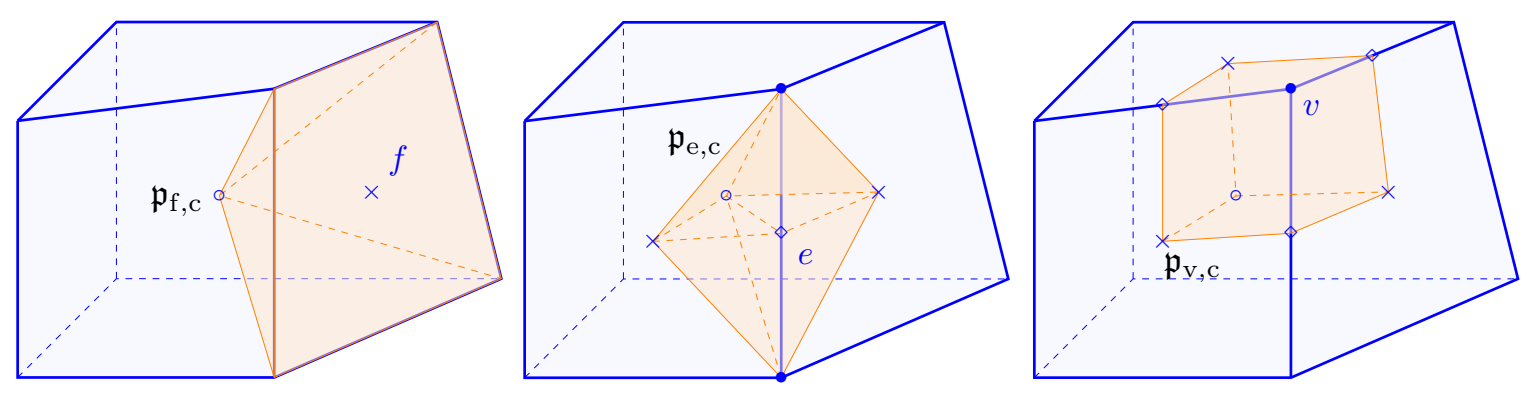

Figure 4: Examples of an element of $\mathfrak{P}_{\mathrm{F}, \mathrm{c}}$ (left), $\mathfrak{P}_{\mathrm{E}, \mathrm{c}}$ (middle), and $\mathfrak{P}_{\mathrm{v}, \mathrm{c}}$ (right) in a hexahedral cell.

Definition 21 (Partitions of a cell). We set:

$$
\begin{aligned}
\mathfrak{p}_{\mathrm{v}, \mathrm{c}}:=\bigcup_{\mathrm{e} \in \mathrm{E}_{\mathrm{v}} \cap \mathrm{E}_{\mathrm{c}} \mathrm{f} \in \mathrm{F}_{\mathrm{e}} \cap \mathrm{F}_{\mathrm{c}}} \mathfrak{s}(\mathrm{v}, \mathrm{e}, \mathrm{f}, \overline{\mathrm{c}}), & & \forall \mathrm{v} \in \mathrm{V}_{\mathrm{c}}, \\
\left.\mathfrak{p}_{\mathrm{e}, \mathrm{c}}:=\bigcup_{\mathrm{f} \in \mathrm{F}_{\mathrm{e}} \cap \mathrm{F}_{\mathrm{c}} \mathrm{v} \in \mathrm{V}_{\mathrm{e}}} \bigcup_{\mathfrak{p}_{\mathrm{f}, \mathrm{c}}}:=\bigcup_{\mathrm{e} \in \mathrm{E}_{\mathrm{f}}} \bigcup_{\mathrm{v} \in \mathrm{V}_{\mathrm{f}}} \mathfrak{s}(\mathrm{v}, \mathrm{e}, \mathrm{f}, \overline{\mathrm{c}}), \overline{\mathrm{c}}\right), & & \forall \mathrm{e} \in \mathrm{E}_{\mathrm{c}}
\end{aligned}
$$

The vertex-based partition is denoted by $\mathfrak{P}_{\mathrm{v}, \mathrm{c}}:=\left\{\mathfrak{p}_{\mathrm{v}, \mathrm{c}}\right\}_{\mathrm{v} \in \mathrm{V}_{\mathrm{c}}}$, the edge-based partition by $\mathfrak{P}_{\mathrm{E}, \mathrm{c}}:=\left\{\mathfrak{p}_{\mathrm{e}, \mathrm{c}}\right\}_{\mathrm{e} \in \mathrm{E}_{\mathrm{c}}}$, and the face-based partition by $\mathfrak{P}_{\mathrm{F}, \mathrm{c}}:=\left\{\mathfrak{p}_{\mathrm{f}, \mathrm{c}}\right\}_{\mathrm{f} \in \mathrm{F}_{\mathrm{c}}}$; see Figure 4 .

Remark 22 (Case $\mathrm{X}=\mathrm{C}$ ). Applying the same rationale as in Definition 21 leads to $\mathfrak{p}_{\mathrm{c}, \mathrm{c}}:=\mathrm{c}$.

Remark 23 (link between $\mathfrak{p}_{\mathrm{v}, \mathrm{c}}$ and $\tilde{\mathfrak{g}}_{\mathrm{V}_{\mathrm{c}}}(\mathrm{v})$ ). By definition, $\left|\mathfrak{p}_{\mathrm{v}, \mathrm{c}}\right|=\tilde{\mathfrak{g}}_{\mathrm{V}_{\mathrm{c}}}(\mathrm{v})$ for each vertex $\mathrm{v} \in \mathrm{V}_{\mathrm{c}}$. 
hold:

We readily verify that $\sum_{\mathrm{x} \in \mathrm{X}_{\mathrm{c}}}\left|\mathfrak{p}_{\mathrm{x}, \mathrm{c}}\right|=|\mathrm{c}|$ for each cell $\mathrm{c} \in \mathrm{C}$. Observe also that the followings identities

$$
\begin{aligned}
\left|\mathfrak{p}_{\mathrm{v}, \mathrm{c}}\right| & =\tilde{\mathfrak{g}}_{\mathrm{V}_{\mathrm{c}}}(\mathrm{v}), & & \forall \mathrm{v} \in \mathrm{V}_{\mathrm{c}} \\
\left|\mathfrak{p}_{\mathrm{e}, \mathrm{c}}\right| & =\frac{1}{d} \mathfrak{g}_{\mathrm{E}_{\mathrm{c}}}(\mathrm{e}) \cdot \tilde{\mathfrak{g}}_{\mathrm{E}_{\mathrm{c}}}(\mathrm{e}), & & \forall \mathrm{e} \in \mathrm{E}_{\mathrm{c}} \\
\left|\mathfrak{p}_{\mathrm{f}, \mathrm{c}}\right| & =\frac{1}{d} \mathfrak{g}_{\mathrm{F}_{\mathrm{c}}}(\mathrm{f}) \cdot \tilde{\mathfrak{g}}_{\mathrm{F}_{\mathrm{c}}}(\mathrm{f}), & & \forall \mathrm{f} \in \mathrm{F}_{\mathrm{c}}
\end{aligned}
$$

\subsection{Generic definition}

Definition 24 (Piecewise constant reconstructions). We set $\mathrm{L}_{\mathcal{X}_{\mathrm{c}}}=\mathrm{C}_{\mathcal{X}_{\mathrm{c}}}+\mathrm{S}_{\mathcal{X}_{\mathrm{c}}}$ with $\mathrm{C}_{\mathcal{X}_{\mathrm{c}}}$ defined by (18) and $\mathrm{S}_{\mathcal{X}_{\mathrm{c}}}: \mathcal{X}_{\mathrm{c}} \rightarrow \mathbb{P}_{0}\left(\mathfrak{P}_{\mathrm{X}, \mathrm{c}}\right)$ (the space spanned by $\mathbb{E}_{\mathrm{X}}$-valued constant fields in each $\left.\mathfrak{p}_{\mathrm{x}, \mathrm{c}}\right)$ defined for all $\mathbf{a} \in \mathcal{X}_{\mathrm{c}}$ as follows:

$$
\mathrm{S}_{\mathcal{X}_{\mathrm{c}}}(\mathbf{a}):=\hat{\mathrm{S}}_{\mathcal{X}_{\mathrm{c}}}\left(\mathbf{a}-\mathrm{R}_{\mathcal{X}_{\mathrm{c}}} \mathrm{C}_{\mathcal{X}_{\mathrm{c}}}(\mathbf{a})\right)
$$

where for all $\mathbf{b} \in \mathcal{X}_{\mathrm{c}}$,

$$
\left.\hat{\mathrm{S}}_{\mathcal{X}_{\mathrm{c}}}(\mathbf{b})\right|_{\mathfrak{p}_{\mathrm{x}, \mathrm{c}}}:=\beta \frac{\tilde{\mathfrak{g}}_{\mathrm{x}_{\mathrm{c}}}(\mathrm{x})}{\left|\mathfrak{p}_{\mathrm{x}, \mathrm{c}}\right|} \mathbf{b}_{\mathrm{x}}, \quad \forall \mathrm{x} \in \mathrm{X}_{\mathrm{c}}
$$

$\beta>0$ is a free-parameter related to the stabilization.

In terms of reconstruction functions, the stabilization part corresponding to Definition 24 is defined as follows:

$$
\left.\ell_{\mathrm{x}, \mathrm{c}}^{\mathrm{St}}\right|_{\mathfrak{p}_{\mathrm{x}^{\prime}, \mathrm{c}}}:=\beta \frac{\tilde{\mathfrak{g}}_{\mathrm{x}_{\mathrm{c}}}(\mathrm{x})}{\left|\mathfrak{p}_{\mathrm{x}^{\prime}, \mathrm{c}}\right|}\left(\delta_{\mathrm{x}, \mathrm{x}^{\prime}}-\frac{\tilde{\mathfrak{g}}_{\mathrm{x}_{\mathrm{c}}}\left(\mathrm{x}^{\prime}\right) \otimes \mathfrak{g}_{\mathrm{x}_{\mathrm{c}}}\left(\mathrm{x}^{\prime}\right)}{|\mathrm{c}|}\right)
$$

The circulation and flux reconstruction operators proposed in DGA schemes correspond to the choice $\beta=\frac{1}{d}$, while the circulation reconstruction operator proposed in HFV schemes corresponds to the choice $\beta=\frac{1}{\sqrt{d}}$.

Proposition 25. Assume (MB). Then, $\mathrm{S}_{\mathcal{X}_{\mathrm{c}}}$ specified in Definition 24 verifies properties $(\mathbf{R} 2 *)$ and $(\mathbf{R} 3 *)$.

Proof. (R2 $*$ ) is a straightforward consequence of (19) and (28). Let us now verify (R3*). Starting from (30), we infer that $\int_{\mathrm{c}} \ell_{\mathrm{x}, \mathrm{c}}^{\mathrm{St}}=\left.\sum_{\mathrm{x}^{\prime} \in \mathrm{X}_{\mathrm{c}}} \int_{\mathfrak{p}_{\mathrm{x}^{\prime}, \mathrm{c}}} \ell_{\mathrm{x}, \mathrm{c}}\right|_{\mathfrak{x}_{\mathrm{x}^{\prime}, \mathrm{c}}}=\beta \tilde{\mathfrak{g}}_{\mathrm{x}_{\mathrm{c}}}(\mathrm{x})-\beta \frac{\tilde{\mathfrak{g}}_{\mathrm{c}}(\mathrm{x})}{|\mathrm{c}|} \sum_{\mathrm{x}^{\prime} \in \mathrm{X}_{\mathrm{c}}} \tilde{\mathfrak{g}}_{\mathrm{x}_{\mathrm{c}}}\left(\mathrm{x}^{\prime}\right) \otimes \mathfrak{g}_{\mathrm{x}_{\mathrm{c}}}\left(\mathrm{x}^{\prime}\right)=0$, owing to (7) for the last identity.

\subsection{Specific definitions}

Potential reconstruction operators. $\quad L_{\mathcal{V}_{\mathrm{c}}}: \mathcal{V}_{\mathrm{c}} \rightarrow \mathbb{P}_{0}\left(\mathfrak{P}_{\mathrm{v}, \mathrm{c}}\right)$ is defined for all $\mathbf{p} \in \mathcal{V}_{\mathrm{c}}$ from the two following contributions:

$$
\mathrm{C}_{\mathcal{V}_{\mathrm{c}}}(\mathbf{p}):=\frac{1}{|\mathrm{c}|} \sum_{\mathrm{v} \in \mathrm{V}_{\mathrm{c}}} \tilde{\mathfrak{g}}_{\mathrm{V}_{\mathrm{c}}}(\mathrm{v}) \mathbf{p}_{\mathrm{v}}
$$

and, for all $\mathrm{v}^{\prime} \in \mathrm{V}_{\mathrm{c}}$,

$$
\left.\mathrm{S}_{\mathcal{V}_{\mathrm{c}}}(\mathbf{p})\right|_{\mathfrak{p}_{\mathrm{v}^{\prime}, \mathrm{c}}}=\frac{\beta}{|\mathrm{c}|} \sum_{\mathrm{v} \in \mathrm{V}_{\mathrm{c}}} \tilde{\mathfrak{g}}_{\mathrm{v}_{\mathrm{c}}}(\mathrm{v})\left(\mathbf{p}_{\mathrm{v}^{\prime}}-\mathbf{p}_{\mathrm{v}}\right)
$$

In terms of reconstruction functions, (31) yields

$$
\begin{array}{ll}
\ell_{\mathrm{v}, \mathrm{c}}^{\mathrm{Co}}:=\frac{\tilde{\mathfrak{g}}_{\mathrm{v}_{\mathrm{c}}}(\mathrm{v})}{|\mathrm{c}|}, & \forall \mathrm{v} \in \mathrm{V}_{\mathrm{c}}, \\
\left.\ell_{\mathrm{v}, \mathrm{c}}^{\mathrm{St}}\right|_{\mathfrak{p}_{\mathrm{v}^{\prime}, \mathrm{c}}}=\beta \frac{\tilde{\mathfrak{g}}_{\mathrm{v}_{\mathrm{c}}}(\mathrm{v})}{|\mathrm{c}|}\left(\delta_{\mathrm{v}, \mathrm{v}^{\prime}}-1\right), & \forall \mathrm{v}, \mathrm{v}^{\prime} \in \mathrm{V}_{\mathrm{c}} .
\end{array}
$$

We observe that the value of these functions is not necessarily continuous across the faces of the partition (induced by $\mathfrak{P}_{\mathrm{v}, \mathrm{c}}$ ) lying inside $\mathrm{c}$, so that, in general, $\mathrm{L} \mathcal{V}_{\mathrm{c}}$ does not map into $H^{1}(\mathrm{c})$. 
Circulation reconstruction operator. $\underline{L}_{\mathcal{E}_{\mathrm{c}}}: \mathcal{E}_{\mathrm{c}} \rightarrow\left[\mathbb{P}_{0}\left(\mathfrak{P}_{\mathrm{E}, \mathrm{c}}\right)\right]^{3}$ is defined for all $\mathbf{u} \in \mathcal{E}_{\mathrm{c}}$ from the two following contributions:

$$
\underline{\mathcal{C}}_{\mathcal{E}_{\mathrm{c}}}(\mathbf{u}):=\frac{1}{|\mathrm{c}|} \sum_{\mathrm{e} \in \mathrm{E}_{\mathrm{c}}} \mathbf{u}_{\mathrm{e}} \tilde{\mathfrak{g}}_{\mathrm{E}_{\mathrm{c}}}(\mathrm{e})
$$

and, for all $\mathrm{e}^{\prime} \in \mathrm{E}_{\mathrm{c}}$,

$$
\left.\underline{\mathrm{S}}_{\mathcal{E}_{\mathrm{c}}}(\mathbf{u})\right|_{\mathfrak{p}_{\mathrm{e}^{\prime}, \mathrm{c}}}:=\beta \frac{\tilde{\mathfrak{g}}_{\mathrm{E}_{\mathrm{c}}}\left(\mathrm{e}^{\prime}\right)}{\left|\mathfrak{p}_{\mathrm{e}^{\prime}, \mathrm{c}}\right|}\left(\mathbf{u}_{\mathrm{e}^{\prime}}-\mathfrak{g}_{\mathrm{E}_{\mathrm{c}}}\left(\mathrm{e}^{\prime}\right) \cdot \underline{\mathrm{C}}_{\mathcal{E}_{\mathrm{c}}}(\mathbf{u})\right) .
$$

In terms of reconstruction functions, (33) yields

$$
\ell_{\mathrm{e}, \mathrm{c}}^{\mathrm{Co}}:=\frac{\tilde{\mathfrak{g}}_{\mathrm{E}_{\mathrm{c}}}(\mathrm{e})}{|\mathrm{c}|}, \quad \forall \mathrm{e} \in \mathrm{E}_{\mathrm{c}}
$$

and, for all $\mathrm{e}, \mathrm{e}^{\prime} \in \mathrm{E}_{\mathrm{c}}$,

$$
\left.\ell_{\mathrm{e}, \mathrm{c}}^{\mathrm{St}}\right|_{\mathfrak{p}_{\mathrm{e}^{\prime}, \mathrm{c}}}=\beta\left(\delta_{\mathrm{e}, \mathrm{e}^{\prime}}-\frac{\tilde{\mathfrak{g}}_{\mathrm{E}_{\mathrm{c}}}\left(\mathrm{e}^{\prime}\right) \otimes \mathfrak{g}_{\mathrm{E}_{\mathrm{c}}}\left(\mathrm{e}^{\prime}\right)}{|\mathrm{c}|}\right) \frac{\tilde{\mathfrak{g}}_{\mathrm{E}_{\mathrm{c}}}(\mathrm{e})}{\left|\mathfrak{p}_{\mathrm{e}^{\prime}, \mathrm{c}}\right|} .
$$

We observe that the tangential component of these functions is not necessarily continuous on the edges of the submesh (induced by $\mathfrak{P}_{\mathrm{E}, \mathrm{c}}$ ) lying inside $\mathrm{c}$, so that, in general, $\underline{\underline{L}}_{\mathcal{E}_{\mathrm{c}}}$ does not map into $H$ (curl; $\mathrm{c}$ ).

Flux reconstruction operator. $\underline{\underline{L}}_{\mathcal{F}_{\mathrm{c}}}: \mathcal{F}_{\mathrm{c}} \rightarrow\left[\mathbb{P}_{0}\left(\mathfrak{P}_{\mathrm{F}, \mathrm{c}}\right)\right]^{3}$ is defined for all $\phi \in \mathcal{F}_{\mathrm{c}}$ from the two following contributions:

$$
\underline{\mathrm{C}}_{\mathcal{F}_{\mathrm{c}}}(\phi):=\frac{1}{|\mathrm{c}|} \sum_{\mathrm{f} \in \mathrm{F}_{\mathrm{c}}} \phi_{\mathrm{f}} \tilde{\mathfrak{g}}_{\mathrm{F}_{\mathrm{c}}}(\mathrm{f}),
$$

and, for all $\mathrm{f}^{\prime} \in \mathrm{F}_{\mathrm{c}}$,

$$
\left.\underline{\mathbf{S}}_{\mathcal{F}_{\mathrm{c}}}(\phi)\right|_{\mathfrak{p}_{\mathfrak{f}^{\prime}, c}}:=\beta \frac{\tilde{\mathfrak{g}}_{\mathrm{F}_{\mathrm{c}}}\left(\mathrm{f}^{\prime}\right)}{\left|\mathfrak{p}_{\mathrm{f}^{\prime}, \mathrm{c}}\right|}\left(\phi_{\mathrm{f}^{\prime}}-\mathfrak{g}_{\mathrm{F}_{\mathrm{c}}}\left(\mathrm{f}^{\prime}\right) \cdot \underline{\mathrm{C}}_{\mathcal{F}_{\mathrm{c}}}(\phi)\right) .
$$

In terms of reconstruction functions, (35) yields

$$
\ell_{\mathrm{f}, \mathrm{c}}^{\mathrm{Co}}:=\frac{\tilde{\mathfrak{g}}_{\mathrm{F}_{\mathrm{c}}}(\mathrm{f})}{|\mathrm{c}|}, \quad \forall \mathrm{f} \in \mathrm{F}_{\mathrm{c}},
$$

and, for all $\mathrm{f}, \mathrm{f}^{\prime} \in \mathrm{F}_{\mathrm{c}}$,

$$
\left.\ell_{\mathrm{f}, \mathrm{c}}^{\mathrm{St}}\right|_{\mathfrak{p}_{\mathrm{f}^{\prime}, \mathrm{c}}}=\beta\left(\delta_{\mathrm{f}, \mathrm{f}^{\prime}}-\frac{\tilde{\mathfrak{g}}_{\mathrm{F}_{\mathrm{c}}}\left(\mathrm{f}^{\prime}\right) \otimes \mathfrak{g}_{\mathrm{F}_{\mathrm{c}}}\left(\mathrm{f}^{\prime}\right)}{|\mathrm{c}|}\right) \frac{\tilde{\mathfrak{g}}_{\mathrm{F}_{\mathrm{c}}}(\mathrm{f})}{\left|\mathfrak{p}_{\mathrm{f}^{\prime}, \mathrm{c}}\right|} .
$$

We observe that the normal component of these functions is not necessarily continuous accross the faces of

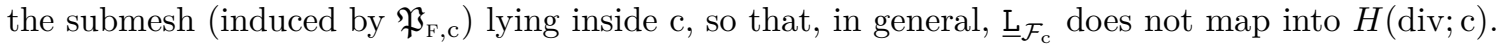

Proposition 26 (Unisolvence). $\mathrm{L}_{\mathcal{X}_{\mathrm{c}}}$ defined from Definition 24 verifies $(\mathbf{R 4})$ if and only if

$$
\beta=1 \text { if } \mathcal{X}_{\mathrm{c}}=\mathcal{V}_{\mathrm{c}} \text { and } \beta=\frac{1}{d} \text { if } \mathcal{X}_{\mathrm{c}} \in\left\{\mathcal{E}_{\mathrm{c}}, \mathcal{F}_{\mathrm{c}}\right\} \text {. }
$$

Proof. The case $\mathcal{V}_{\mathrm{c}}$ is readily verified starting from (32). The case $\mathcal{E}_{\mathrm{c}}$ stems from (27b). For all edges e $\in \mathrm{E}_{\mathrm{c}}$, the following identity holds

$$
\int_{\mathrm{e}} \underline{\ell}_{\mathrm{e}, \mathrm{c}} \cdot \underline{\tau}_{\mathrm{e}}=\tilde{\mathfrak{g}}_{\mathrm{E}_{\mathrm{c}}}(\mathrm{e}) \cdot \tilde{\mathfrak{g}}_{\mathrm{E}_{\mathrm{c}}}(\mathrm{e})\left(\frac{1}{|\mathrm{c}|}+\frac{\beta}{\left|\mathfrak{p}_{\mathrm{e}, \mathrm{c}}\right|}-\frac{\beta \tilde{\mathfrak{g}}_{\mathrm{E}_{\mathrm{c}}}(\mathrm{e}) \cdot \tilde{\mathfrak{g}}_{\mathrm{E}_{\mathrm{c}}}(\mathrm{e})}{\left|\mathfrak{p}_{\mathrm{e}, \mathrm{c}}\right||\mathrm{c}|}\right)=1+(\beta d-1)\left(1-\frac{d\left|\mathfrak{p}_{\mathrm{e}, \mathrm{c}}\right|}{|\mathrm{c}|}\right),
$$

and the right-hand side equals 1 if and only if $\beta=\frac{1}{d}$. The proof for the case $\mathcal{F}_{\mathrm{c}}$ follows the same lines.

Remark 27. The choice $\beta=\frac{1}{\sqrt{d}}$ adopted in HFV schemes has the practical advantage to yield a diagonal discrete Hodge operator when the material property is isotropic and the mesh is superadmissible (see Eymard et al., 2010, Lemma 2.1). On the other hand, adapting the arguments of (Di Pietro 8 Lemaire, 2015, Lemma 8), the choice $\beta=\frac{1}{d}$ allows one to devise a piecewise affine potential reconstruction on the pyramidal submesh $\bigcup_{\mathrm{c} \in \mathrm{C}} \mathfrak{P}_{\mathrm{F}, \mathrm{c}}$ with continuous mean values at interfaces of the submesh. 


\section{Application}

\subsection{CDO schemes for diffusion problems}

In this section, we focus on CDO vertex-based schemes for elliptic problems as introduced in Bonelle \& Ern (2014a). The model problem is

$$
-\operatorname{div}(\underline{\underline{\kappa}} \underline{\operatorname{grad}}(p))=s \quad \text { in } \Omega
$$

where $p$ is termed the potential, $\underline{\underline{\kappa}}$ the conductivity tensor (assumed to be symmetric with eigenvalues uniformly bounded from above and from below away from zero), and $s$ the source term. We consider Dirichlet boundary conditions. The discrete system is: Find $\mathbf{p} \in \mathcal{V}$ such that, for all $\mathbf{q} \in \mathcal{V}$,

$$
\mathrm{H}_{\kappa}^{\mathcal{E}}(\operatorname{GRAD}(\mathbf{p}), \operatorname{GRAD}(\mathbf{q}))=\int_{\Omega} s \mathrm{~L}_{\mathcal{V}}^{0}(\mathbf{q})
$$

$\mathrm{L}_{\mathcal{V}}^{0}$ is defined as the piecewise constant reconstruction detailed in (31) with the choice $\beta=1$. The global Hodge inner product is simply defined by collecting the local contributions $\mathrm{H}_{\kappa}^{\mathcal{E}}(\mathbf{u}, \mathbf{v}):=\sum_{\mathrm{c} \in \mathrm{C}} \mathrm{H}_{\kappa}^{\mathcal{E}_{\mathrm{c}}}\left(\mathbf{u}_{\mathrm{c}}, \mathbf{v}_{\mathrm{c}}\right)$ where $\mathbf{u}_{\mathrm{c}}$ and $\mathbf{v}_{\mathrm{c}}$ are the restriction of the global DoFs to the cell $\mathrm{c} \in \mathrm{C}$, i.e. $\mathbf{u}_{\mathrm{c}}, \mathbf{v}_{\mathrm{c}} \in \mathcal{E}_{\mathrm{c}}$. The discrete gradient operator GRAD $: \mathcal{V} \rightarrow \mathcal{E}$ is defined as follows:

$$
\left.\operatorname{GRAD}(\mathbf{p})\right|_{\mathrm{e}}=\sum_{\mathrm{v} \in \mathrm{V}_{\mathrm{e}}} \iota_{\mathrm{v}, \mathrm{e}} \mathbf{p}_{\mathrm{v}}, \quad \forall \mathrm{e} \in \mathrm{E},
$$

with the incidence number is such that $\iota_{\mathrm{v}, \mathrm{e}}=1$ if $\underline{\tau}_{\mathrm{e}}$ points towards $\mathrm{v}, \iota_{\mathrm{v}, \mathrm{e}}=-1$ otherwise.

\subsection{Numerical results}

We consider the first test case of the FVCA benchmark Eymard et al. (2011). The domain $\Omega$ is the unit cube $[0,1]^{3}$, and the exact potential and the conductivity are

$$
\begin{aligned}
& p(x, y, z):=1+\sin (\pi x) \sin \left(\pi\left(y+\frac{1}{2}\right)\right) \sin \left(\pi\left(z+\frac{1}{3}\right)\right), \\
& \underline{\underline{\kappa}}:=\left[\begin{array}{ccc}
1 & 0.5 & 0 \\
0.5 & 1 & 0.5 \\
0 & 0.5 & 1
\end{array}\right]
\end{aligned}
$$

The source term and the Dirichlet boundary conditions are set according to (40). Since the global linear system is SPD by construction, it can be efficiently solved using a preconditioned Conjugate Gradient method. Two sequences of three-dimensional polyhedral meshes are tested, each family consisting of successive uniform refinements of an initial mesh. The first mesh sequence, hereafter denoted by PrG, contains prismatic cells with polygonal basis, and the second one, hereafter denoted by CB, checkerboard cells with hanging nodes; see Figure 5. The finest mesh of the PrG sequence contains approximately 150,000 vertices and 350,000 edges and that of the CB sequence 250,000 vertices and 700,000 edges.
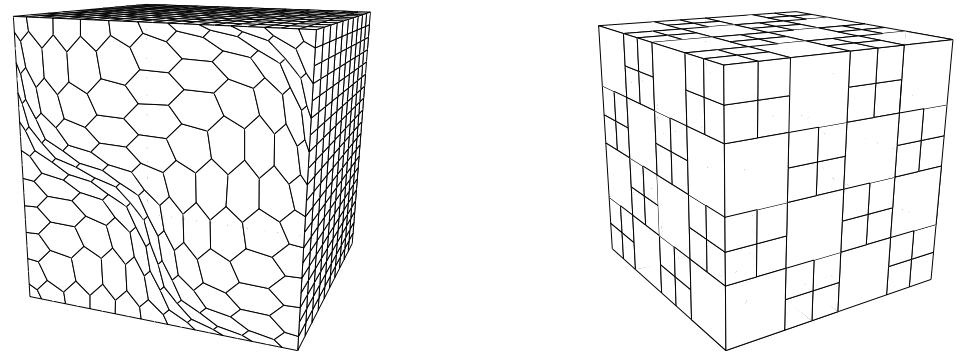

Figure 5: Two examples of polyhedral meshes. Left: prismatic mesh with a polygonal basis; Right: checkerboard mesh with hanging nodes. 
Accuracy. We perform a comparative study of the reconstruction operators by computing a discrete error on the potential $\operatorname{Er}_{\mathcal{V}}(\mathbf{p})$ and a discrete energy error on the gradient $\mathbf{E r}_{\mathcal{E}}(\mathbf{g})$ defined as follows:

$$
\begin{aligned}
& \operatorname{Er}_{\mathcal{V}}(\mathbf{p}):=\frac{\left\|\mathrm{R}_{\mathcal{V}}(p)-\mathbf{p}\right\| \mathcal{V}}{\left\|\mathrm{R}_{\mathcal{V}}(p)\right\| \mathcal{V}}, \\
& \operatorname{Er}_{\mathcal{E}}(\mathbf{g}):=\sqrt{\frac{\mathbf{H}_{\mathcal{K}}^{\mathcal{E}}\left(\mathrm{R}_{\mathcal{E}}(\underline{g})-\mathbf{g}, \mathrm{R}_{\mathcal{E}}(\underline{g})-\mathbf{g}\right)}{\mathrm{H}_{\mathcal{K}}^{\mathcal{E}}\left(\mathrm{R}_{\mathcal{E}}(\underline{g}), \mathrm{R}_{\mathcal{E}}(\underline{g})\right)}},
\end{aligned}
$$

where $g:=\operatorname{grad}(p)$ and $\mathbf{g}:=\operatorname{GRAD}(\mathbf{p})$.

We plot the error $\operatorname{Er}_{\mathcal{V}}(\mathbf{p})$ and the error $\operatorname{Er}_{\mathcal{E}}(\mathbf{g})$ in Figure 6. Four values of the stabilization parameter $\beta$ are considered: an under-penalized value $\left(\frac{1}{d^{3}}\right)$ the one used in DGA $\left(\frac{1}{d}\right)$, the one used in $\operatorname{HFV}\left(\frac{1}{\sqrt{d}}\right)$, and an over-penalized value $(d)$. Labels associated with each case are collected in Table 1.

\begin{tabular}{lcccc}
\hline$\beta$ & $\frac{1}{d^{3}}$ & $\frac{1}{d}$ & $\frac{1}{\sqrt{d}}$ & $d$ \\
\hline $\operatorname{PrG}$ & - & $\multimap$ & $-\bullet-$ & $\cdots . \square \cdots$ \\
$\mathrm{CB}$ & - & $\multimap$ & $-\bullet-$ & $\cdots \oplus \cdots$ \\
\hline
\end{tabular}

Table 1: Labels associated with each case ( $\beta$, mesh sequence).

We observe that the over-penalized scheme produces a larger error. We also notice a super-convergence in the energy norm for PrG meshes, as already observed by Bonelle \& Ern (2014a). In Figure 7, we plot the error
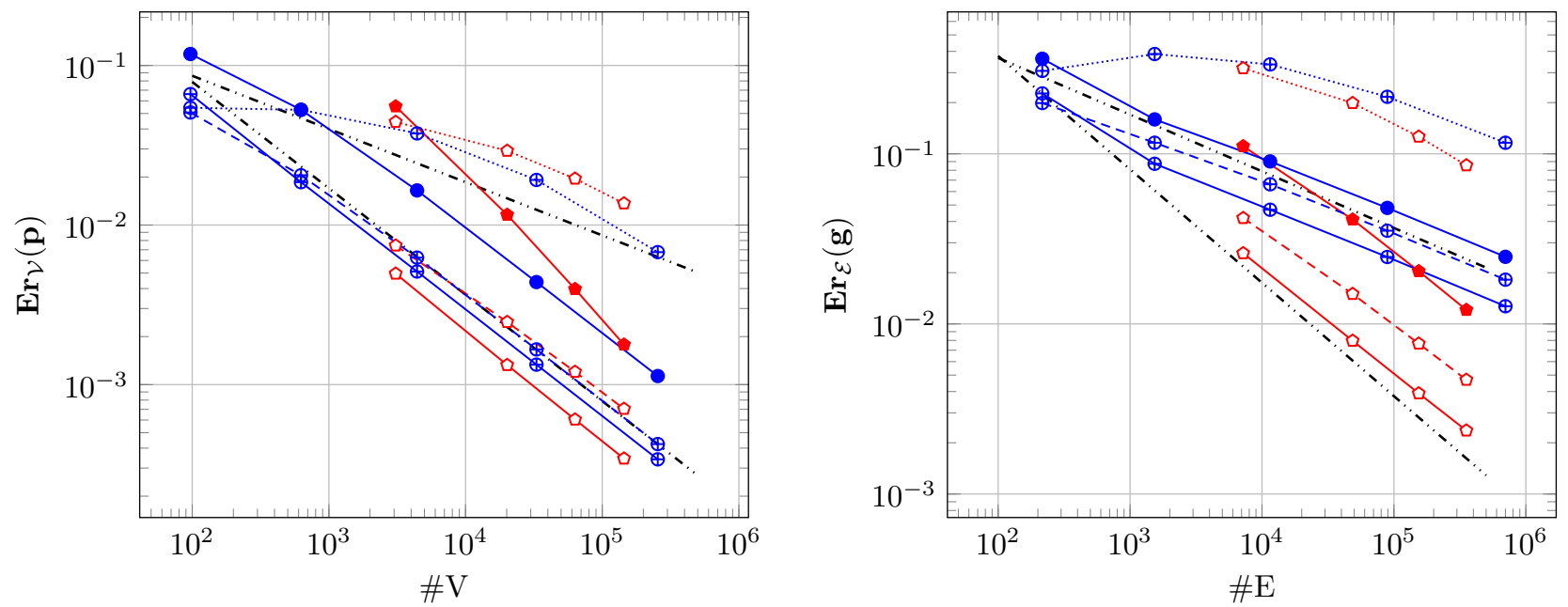

Figure 6: Left: Discrete error on the potential for different values of $\beta$ and the two mesh sequences. Right: Discrete error on the gradient for different values of $\beta$ and the two mesh sequences. Dash-dotted lines indicating first- and second-order convergence rates are included.

$\operatorname{Er}_{\mathcal{E}}(\mathrm{g})$ for a large set of values of $\beta$ and for the $\operatorname{PrG}$ and $\mathrm{CB}$ mesh sequences. Values of $\beta$ around $\frac{1}{d}$ yield the most accurate results for the current test cases.

Cost. In order to compare the efficiency to solve the linear systems produced by the different reconstruction operators, we define the computational cost $\chi:=N N Z \times n_{\text {Ite }}$, where NNZ is the number of nonzero entries of the matrix to invert and $\mathrm{n}_{\text {tte }}$ is the number of iterations performed by the iterative solver to reduce the Euclidean norm of the residual below a tolerance set at $10^{-12} \cdot \chi$ provides a reasonable estimate of the computational cost to solve the linear system since the most costly operation in an iterative solver such as the Conjugate Gradient is the matrix-vector product. In Figure 8, we plot the computational cost $\chi$ for a large set of values of $\beta$ and 

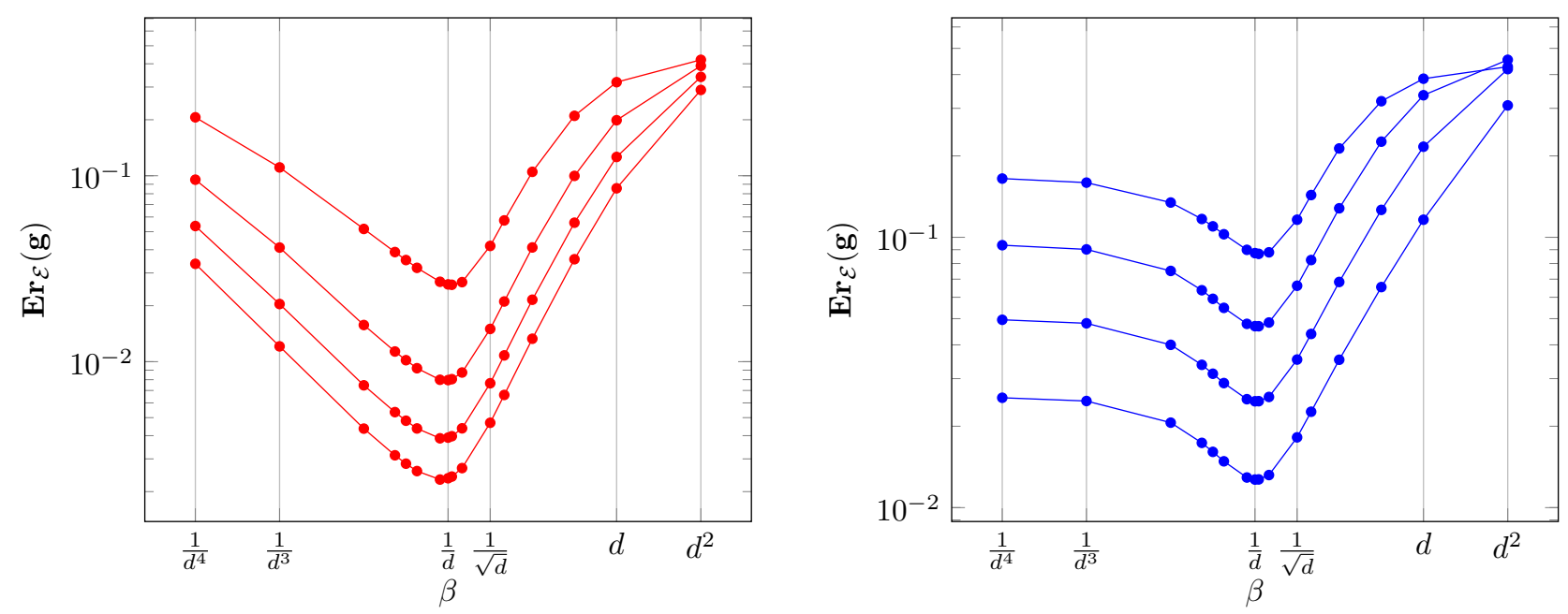

Figure 7: Error $\operatorname{Er}_{\mathcal{E}}(\mathbf{g})$ as a function of $\beta$ for each mesh of the $\operatorname{PrG}$ sequence (right) and of the CB sequence (right).
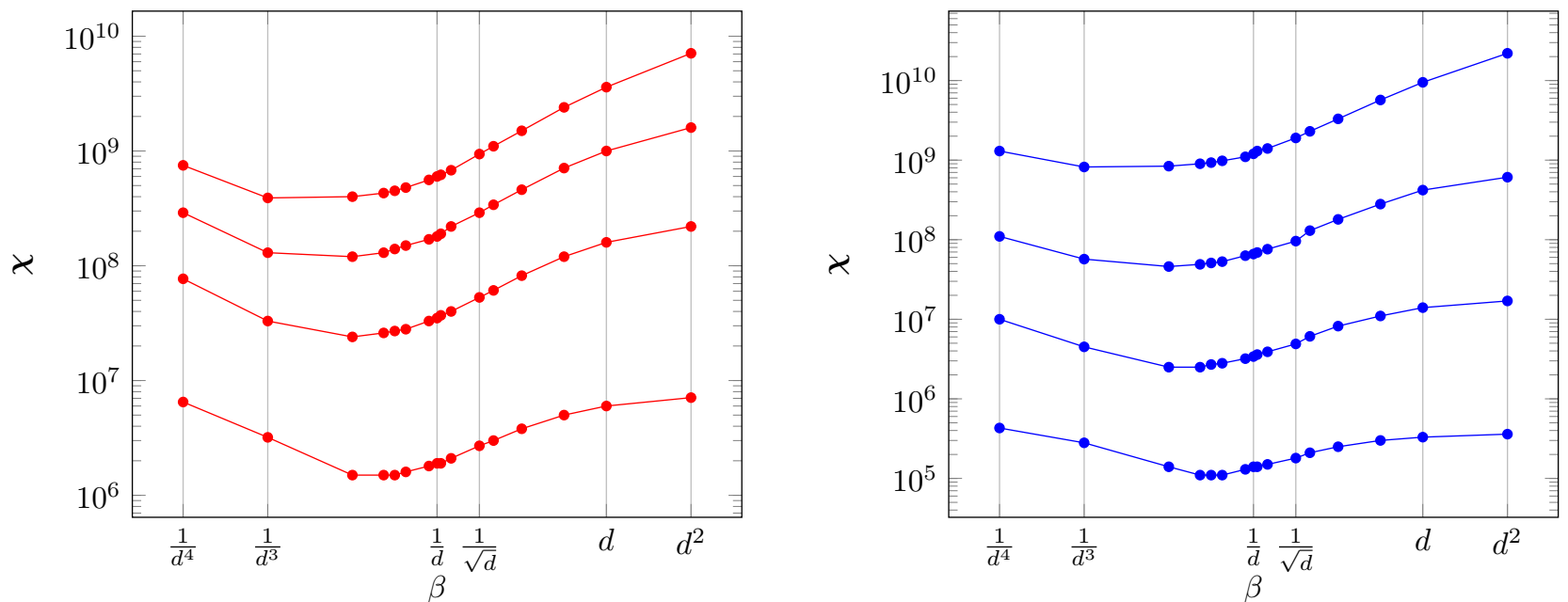

Figure 8: Computational cost $\chi$ as a function of $\beta$ for each mesh of the PrG sequence (left) and the CB sequence (right).

for the PrG and CB mesh sequences. The computational cost is higher for the schemes with an over-penalized value of $\beta$, and it is also slightly higher for an under-penalized value.

Preservation of bounds. Finally, we investigate numerically the discrete minimum/maximum principle $(\mathrm{DMP})$. Setting $\mathbf{p}_{\min }:=\min _{\mathrm{v} \in \mathrm{V}} \mathbf{p}_{\mathrm{v}}$ and $\mathbf{p}_{\max }:=\max _{\mathrm{v} \in \mathrm{V}} \mathbf{p}_{\mathrm{v}}$, we consider that the discrete minimum (resp. maximum) principle is numerically satisfied if $\mathbf{p}_{\min } \geq \min _{x \in \bar{\Omega}} p(\underline{x})$ (resp. $\mathbf{p}_{\max } \leq \max _{x \in \bar{\Omega}} p(\underline{x})$ ). Results are collected in Table 2. Y indicates that the DMP is satisfied (minimum or maximum) for all the meshes of the sequence and $\mathrm{N}$ indicates that at least one mesh in the sequence does not respect the criterion. Using an under-penalized value of $\beta$ negatively impacts the DMP.

\section{Conclusion}

In this work, we have studied low-order reconstruction operators for polyhedral meshes in a unified framework for degrees of freedom attached to vertices, edges, faces, and cells. These reconstruction operators provide a systematic way of building a Hodge inner product which is a key concept for the compatible numerical 


\begin{tabular}{llcccccc}
\hline \multirow{2}{*}{ Mesh } & $\beta$ & $\frac{1}{d^{4}}$ & $\frac{1}{d^{3}}$ & $\frac{1}{d}$ & $\frac{1}{\sqrt{d}}$ & $d$ & $d^{2}$ \\
\hline \multirow{2}{*}{ PrG } & $\min$ & $\mathrm{N}$ & $\mathrm{N}$ & $\mathrm{Y}$ & $\mathrm{Y}$ & $\mathrm{Y}$ & $\mathrm{Y}$ \\
& $\max$ & $\mathrm{N}$ & $\mathrm{N}$ & $\mathrm{Y}$ & $\mathrm{Y}$ & $\mathrm{Y}$ & $\mathrm{Y}$ \\
\hline \multirow{2}{*}{$\mathrm{CB}$} & $\min$ & $\mathrm{N}$ & $\mathrm{Y}$ & $\mathrm{Y}$ & $\mathrm{Y}$ & $\mathrm{Y}$ & $\mathrm{Y}$ \\
& $\max$ & $\mathrm{N}$ & $\mathrm{N}$ & $\mathrm{Y}$ & $\mathrm{Y}$ & $\mathrm{Y}$ & $\mathrm{Y}$ \\
\hline
\end{tabular}

Table 2: Synthesis of results related to the discrete min./max. principle.

approximation of PDEs. We have presented two equivalent sets of design properties. A simple example of piecewise constant reconstruction operators depending on a single stabilization parameter has been detailed, and the influence of this parameter on accuracy and computational costs has been investigated numerically on an anisotropic diffusion problem using CDO vertex-based schemes. Under- and over-penalized values of the stabilization parameter have a negative impact, on the preservation of bounds and on accuracy and costs, respectively. For the problem considered, appropriate choices are the values proposed in DGA and HFV schemes, with the value $\beta=\frac{1}{d}$ appearing as more favorable. These conclusions are to be confirmed by further numerical tests.

\section{References}

Agélas, L., Di Pietro, D. A., Eymard, R. \& Masson, R. (2010) An abstract analysis framework for nonconforming approximations of diffusion problems on general meshes. IJFV International Journal on Finite Volumes, 7, 1-29.

Auchmann, B. \& Kurz, S. (2006) A geometrical defined discrete Hodge operator on simplicial cells. IEEE Transactions on Magnetics, 42, 643.

Bochev, P. \& Hyman, J. M. (2005) Principles of mimetic discretizations of differential operators. Compatible Spatial Discretization (D. Arnold, P. Bochev, R. Lehoucq, R. A. Nicolaides \& M. Shashkov eds). The IMA Volumes in mathematics and its applications, vol. 142. Springer, pp. 89-120.

Bonelle, J. (2014) Compatible Discrete Operator schemes on polyhedral meshes for elliptic and Stokes equations. Ph.D. thesis, Université Paris-Est.

Bonelle, J. \& ERn, A. (2014a) Analysis of Compatible Discrete Operator Schemes for Elliptic Problems on Polyhedral Meshes. ESAIM: Mathematical Modelling and Numerical Analysis, 48, 553-581. DOI:10.1051/m2an/2013104.

Bonelle, J. \& ERn, A. (2014b) Analysis of Compatible Discrete Operator schemes for the Stokes equations on polyhedral meshes. IMA J. Numer. Anal.

Bossavit, A. (1988) Whitney forms: a class of Finite Elements for three-dimensional ccomputation in electromagnetism. IEE Proceedings A, 135, 493-500.

Bossavit, A. (1999-2000) Computational electromagnetism and geometry. J. Japan Soc. Appl. Electromagn. \& Mech., 7-8, 150-9 (no 1), 294-301 (no 2), 401-8 (no 3), 102-9 (no 4), 203-9 (no 5), 372-7 (no 6).

Brezzi, F., Lipnikov, K. \& Shashkov, M. (2005) Convergence of the Mimetic Finite Difference method for diffusion problems on polyhedral meshes. SIAM J. Numer. Anal, 43, 1872-1896.

Brezzi, F., Lipnikov, K., Shashkov, M. \& Simoncini, V. (2007) A new discretization methodology for diffusion problems on generalized polyhedral meshes. Computer Methods in Applied Mechanics and Engineering, 196, 3682-3692.

Brezzi, F., Buffa, A. \& Lipnikov, K. (2009) Mimetic Finite Difference for elliptic problem. Mathematical Modelling and Numerical Analysis, 43, 277-295.

Brezzi, F., Buffa, A. \& Manzini, G. (2014) Mimetic scalar products of discrete differential forms. J. Comput. Phys., 257, 1228-1259. 
Christiansen, S. H. (2008) A construction of spaces of compatible differential forms on cellular complexes. Math. Models Methods Appl. Sci., 18, 739-757.

Codecasa, L., Specogna, R. \& Trevisan, F. (2010) A new set of basis functions for the Discrete Geometric Approach. Journal of Computational Physics, 229, 7401-7410.

Codecasa, L. \& Trevisan, F. (2007) Constitutive equations for discrete electromagnetic problems over polyhedral grids. Journal of Computational Physics, 225, 1894-1918.

Desbrun, M., Hirani, A. N., Leok, M. \& Marsden, J. E. (2005) Discrete Exterior Calculus. http://arxiv.org/abs/math/0508341.

Desbrun, M., Kanso, E. \& Tong, Y. (2006) Discrete Differential Forms for Computational Modeling. Discrete Differential Forms for Computational Modeling, pp. 287-324.

Di Pietro, D. A., Ern, A. \& Lemaire, S. (2014) An arbitrary-order and compact-stencil discretization of diffusion on general meshes based on local reconstruction operators. Computational Methods in Applied Mathematics, 14, 461-472.

Di Pietro, D. A. \& Ern, A. (2013) A Family of Arbitrary-order Mixed Methods for Heterogeneous Anisotropic Diffusion on General Meshes. http://hal.archives-ouvertes.fr/hal-00918482.

Di Pietro, D. A. \& Ern, A. (2015) A hybrid high-order locking-free method for linear elasticity on general meshes. Comput. Meth. Appl. Mech. Engrg., 283, 1-21.

Di Pietro, D. A. \& Lemaire, S. (2015) An extension of the Crouzeix-Raviart space to general meshes with application to quasi-incompressible linear elasticity and Stokes flow. Math. Comp., 84, 1-31.

Eymard, R., Gallouët, T. \& Herbin, R. (2010) Discretization of heterogeneous and anisotropic diffusion problems on general nonconforming meshes SUSHI: a scheme using stabilization and hybrid interfaces. IMA Journal of Numerical Analysis, 30, 1009-1043.

Eymard, R., Henry, G., Herbin, R., Hubert, F., Klöfkorn, R. \& Manzini, G. (2011) 3D benchmark on discretization schemes for anisotropic diffusion problems on general grids. Finite Volumes for Complex Applications VI - Problems \& Perspectives, vol. 2. Springer, pp. 95-130.

Flonter, M., Kós, G. \& Reimers, M. (2005) Mean value coordinates in 3d. Computer Aided Geometric Design, 22, 623-631.

Frankel, T. (1997) The Geometry of Physics: an Introduction, first edition edn. Cambridge University Press.

Gerritsma, M. (2012) An introduction to a compatible spectral discretization method. Mechanics of Advanced Materials and Structures, 19, 48-67.

Gillette, A., Rand, A. \& BajAJ, C. (2012) Error estimates for generalized barycentric interpolation. Adv. Comput. Math., 37, 417-439.

Gillette, A., Rand, A. \& Bajaj, C. (2014) Construction of scalar and vector finite element families on polygonal and polyhedral meshes. Submitted.

Gillette, A. \& Bajaj, C. (2011) Dual formulations of mixed finite element methods with applications. Computer-Aided Design, 43, 1213 - 1221.

Hiptmair, R. (2001) Discrete Hodge operators: An algebraic perspective. Progress In Electromagnetics Research, 32, 247-269.

Hirani, A. (2003) Discrete Exterior Calculus. Ph.D. thesis, California Institute of Technology.

Hormann, K. \& Sukumar, N. (2008) Maximum entropy coordinates for arbitrary polytopes. Proceedings of the Symposium on Geometry Processing. Eurographics Association, pp. 1513-1520.

Nicolaides, R. A. (1992) Direct discretization of planar div-curl problems. SIAM J. Numer. Ana, 29, $32-56$. 
Tarhasani, T., Kettunen, L. \& Bossavit, A. (1999) Some realizations of a discrete Hodge operator: A reinterpretation of finite element techniques. IEEE Transactions on magnetics, 35, 1494-1497.

TeixeirA, F. (2001) Geometric aspects of the simplicial discretization of Maxwell's equations. Progress In Electromagnetics Research (PIER), 32, 171-188.

Tonti, E. (1975) The reason for analogies between physical theories. Applied Mathematical Modelling, 1, $37-50$.

Wachspress, E. (1975) A rational Finite Element basis. Mathematics in Science and Engineering, vol. 114. Academic Press.

Warren, J., Schaefer, S., Hirani, A. \& Desbrun, M. (2007) Barycentric coordinates for convex sets. Advances in Computational Mathematics, 27, 319-338.

Whitney, H. (1957) Geometric integration theory. Princeton, N. J.: Princeton University Press, pp. xv+387. 